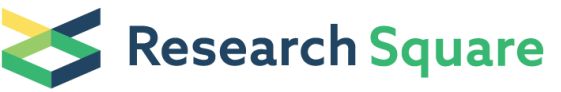 \\ Preprints are preliminary reports that have not undergone peer review. \\ They should not be considered conclusive, used to inform clinical practice, or referenced by the media as validated information.
}

\section{Estimating the Financial Impact of Livestock Schistosomiasis on Traditional Subsistence and Transhumance Farmers Keeping Cattle, Sheep and Goats in Northern Senegal}

Praise Adeyemo ( $\sim$ padeyemo19@rvc.ac.uk)

The Royal Veterinary College Department of Pathobiology and Population Sciences https://orcid.org/0000-0002-28318091

\section{Elsa Léger}

Royal Veterinary College Department of Pathology and Infectious Diseases: The Royal Veterinary College Department of Pathobiology and Population Sciences

\section{Elizabeth Hollenberg}

The Royal Veterinary College Department of Pathobiology and Population Sciences

\section{Nicolas Diouf}

Unité de Formation et de Recherche des Sciences Agronomiques, d'Aquaculture et de Technologies Alimentaires, Université Gaston Berger, Saint-Louis

\section{Mariama Sene}

Universite Gastion Berger Saint Louis UFR de Sciences Agronomiques d'Aquaculture et de Technologie Alimentaire

\section{Joanne P Webster}

London Centre for Neglected Tropical Disease Research, School of Public Health, Imperial College London

\section{Barbara Häsler}

The Royal Veterinary College Department of Pathobiology and Population Sciences

\section{Research Article}

Keywords: Schistosomiasis, One Health, Economics, NTDs, Livestock, Subsistence, Praziquantel, Financial Impact, Partial Budget Analysis, Senegal, Disease control

Posted Date: August 3rd, 2021

DOI: https://doi.org/10.21203/rs.3.rs-757540/v1

License: (c) (i) This work is licensed under a Creative Commons Attribution 4.0 International License. Read Full License

Version of Record: A version of this preprint was published at Parasites \&amp; Vectors on March 22nd, 2022. See the published version at https://doi.org/10.1186/s13071-021-05147-w. 


\section{Abstract}

Background: Schistosomiasis is a disease that poses major threats to human and animal health, as well as the economy, especially in sub-Saharan Africa (SSA). Furthermore, its zoonotic nature and the presence of hybrid species complicate efforts to achieve the new World Health Organization's roadmap for neglected tropical diseases target of elimination. Whilst many studies have evaluated the economic impact of schistosomiasis in humans, only one has been performed to date in livestock in SSA and none in Senegal. The aim of this study was to estimate the financial impact of livestock schistosomiasis in selected regions of Senegal.

Methods: Stochastic partial budget models were developed in RiskAmp add-in for Excel for a one-year period to estimate the disease costs on local traditional farmers in twelve villages from the Lac de Guiers and Barkedji regions, Senegal. Disease costs were the sum of disease losses and expenditures and included reduced income due to production losses (e.g. reduced milk yield), expenditures saved (e.g. concentrate feed saved due to disease), additional costs (e.g. testing and treatment, buying replacement animals), and additional income (e.g. selling of diseased animals). The models were parameterised using primary data from cross-sectional surveys and focus group discussions, as well as secondary data from scientific literature and available statistics. Two scenarios were defined based on the most common practices reported: scenario 1 modelled a situation in which the farmers tested and treated their livestock for schistosomiasis; whilst scenario 2 modelled a situation in which there was no tests nor treatment. The model was run with 10,000 iterations for a period of one year; results were expressed in XOF, i.e., the West African CFA franc (1 XOF is equivalent to 0.0014 GBP) with the median and $95 \%$ confidence range. Sensitivity analyses were conducted to assess the impact of uncertain variables on the output.

Results: For scenario 1, the median disease costs per year and head of cattle, sheep, and goats, respectively, were estimated at XOF -13,408, XOF -27,227 and XOF -27,694. For scenario 2, the disease costs per year and head of cattle, sheep, and goats, respectively, were estimated at XOF -49,296, XOF -70,072 and XOF -70,281. Sensitivity analyses indicated that the market prices for young and adult, healthy and sick animals had the biggest impact on the disease costs for all species.

Conclusions: Our findings suggest that the financial impact of livestock schistosomiasis on traditional subsistence and transhumance farmers in North Senegal is substantial. Consequently, treating livestock schistosomiasis with an effective control strategy has the potential to generate substantial benefits to farmers and their families. Our results can also serve as a baseline for future cost-benefit and cost-effectiveness analyses for potential regional treatment campaigns for schistosomiasis in livestock.

\section{Background}

Schistosomiasis is a major neglected tropical disease (NTD), second only to malaria as a parasitic disease in terms of socio-economic impact [1]. This waterborne NTD, caused by Schistosoma spp dioecious trematodes, affects both humans and animals, being indirectly transmitted to their mammalian definitive hosts via freshwater molluscan intermediate hosts $[2,3,4]$. Over 240 million people are estimated to be infected with schistosomiasis caused by either Schistosoma haematobium (and hybrids therein), S. japonicum, S. mansoni, S. mekongi, S. guineensis or S. intercalatum [5], with over $90 \%$ of human cases occurring within sub-Saharan Africa (SSA) [3].

Whilst the ongoing zoonotic transmission of schistosomiasis between humans and over forty potential mammalian reservoir hosts is fully acknowledged within Asia $[6,7,8]$, there is also an increasingly acknowledged zoonotic role within Africa $[9,10]$, as well as an awareness of the morbidity impact of animal schistosomiasis in general [11, 12]. Although the total number of livestock infected globally has not been accounted for [13], schistosomiasis in domestic animals often occurs with the same underprivileged communities most affected by human schistosomiasis $[9,11]$. In SSA, for example, 
hybrid species such as S. haematobium:S. bovis, S. haematobium:S. curassoni, S. haematobium:S. mattheei and/or $S$. bovis:S. curassoni have been reported in humans, while S. bovis, S. curassoni, S. mattheei, together with S. bovis:S. curassoni and S. bovis: S. mattheeii hybrids have been documented in domestic livestock $[2,14,15,16,17]$.

Since 2002, large-scale mass drug administration (MDA) as preventative chemotherapy with praziquantel (PZQ) of highrisk groups of children, predominantly school-aged children, has been implemented across much of SSA [18]. Morbidity control has been generally successful across many countries [19] and this has helped lead to a revision of the World Health Organization's (WHO's) strategic plan for a vision of "a world free of schistosomiasis" [20, 21], and most recently, the new WHO NTD-Roadmap which aims to achieve elimination as a public health problem (EPHP, i.e. elimination of morbidity where the prevalence of heavy infection intensity less than $1 \%$ in all sentinel sites) in all endemic countries by 2030, as well as a complete interruption of transmission (loT, i.e. reduction of incidence of infection to zero) in selected African regions by the same point [22]. However, the sole focus within SSA of MDA in humans without complementary control of the disease in livestock, as well as misuse of PZQ in animals to control livestock schistosomiasis, continue to frustrate efforts to achieve schistosomiasis control and elimination goals stipulated by the WHO [11].

Furthermore, schistosomiasis has been reported as one of the NTDs with the greatest unequal socioeconomic distribution [23], posing a threat to public health and having grave economic implications $[24,25,26]$. The drug PZQ is donated at a large scale by pharmaceutical companies, predominantly Merck KGA, and given for free to school-aged children across many SSA countries [27] at an estimated value of \$32.5 million annually [28]. Evaluations to date described the cost of the disease in humans in terms of disability-adjusted life years (DALYs), the Quality adjusted life years (QALYs), the number of working days lost, and the financial burden of the disease [25]. Redekop and colleagues [29], for instance, conducted a review of studies on the economic impact of human schistosomiasis in terms of treatment costs and disease costs and estimated the global annual productivity loss associated with schistosomiasis at $\$ 5.5$ billion from $2011-2020$, and \$11.9 billion from 2021-2030.

There is a dearth of studies, in contrast, on the economic implications of the animal form of the disease [11]. A few studies report on the treatment costs for the disease by farmers and the biological effects and productivity impact of livestock schistosomiasis. They found that the different species of schistosomes cause organ pathologies in cattle [ $\left.{ }^{30}\right]$, sheep [31] and goats [32], as well as productivity losses of meat, milk and reproduction [33]. To the authors' knowledge, the only published study estimating the economic impact of schistosomiasis in animals in Africa is a benefit-cost analysis of investing in a potential vaccine for schistosomiasis in cattle in Sudan [33]. In this example, the disease costs considered include production losses, and the capital and operating costs of the vaccination program. The benefit-cost ratio was associated with infection probability, vaccine uptake, mortality and vaccine production costs. The study showed that for every \$1 spent on bovine schistosomiasis in provinces with a high percentage of vaccinated animals and low vaccine production costs, the benefits would be $\$ 12.7$. However, in provinces with low vaccination and high vaccine production costs, the benefit-cost was lower at $\$ 0.7$. The potential benefits identified were related to avoidance of mortality and growth delay losses caused by the disease [33].

The lack of economic assessments of livestock schistosomiasis makes decisions on investment in the treatment of livestock schistosomiasis difficult, particularly given the need to balance any potential benefits gained with that of increased risks in terms of the evolution of PZQ resistance [10], and where there might be other endemic disease priorities for the sector. Livestock schistosomiasis, however, not only affects measures to control or eliminate human schistosomiasis but also causes disease costs for farmers, affects livelihoods and reduces the availability of livestockderived foods for human consumption. Knowledge of the losses caused by disease and expenditures needed for diagnosis and treatment allows generating a baseline of the current impact of the disease [34]. This baseline can then be used in cost-benefit or cost-effectiveness analysis to estimate the potential value of control strategies (e.g. mass or targeted drug treatment of animals) for individual farmers or the sub-sector. 
The aim of this study was to estimate the financial impact of livestock schistosomiasis on traditional subsistence and transhumance farmers in selected villages around the Lac de Guiers and the town of Barkedji in Senegal. The objectives were to: 1) establish herd structures and production parameters for a regular cattle, sheep, and goat herd or flock in North Senegal; and 2) estimate losses and expenditures due to schistosomiasis in these production systems. The findings are discussed in terms of the potential economic impact livestock schistosomiasis can have on the livelihoods of farmers and their communities.

\section{Methods}

\section{Study sites}

This research was carried out in two regions in northern Senegal. Six villages were selected around the town of Barkedji $\left(15.2774^{\circ} \mathrm{N}, 14.8674^{\circ} \mathrm{W}\right)$ in the Linguere department of the Louga region in the Vallée du Ferlo, and six villages around the Lac de Guiers $\left(16.2247^{\circ} \mathrm{N}, 15.8408^{\circ} \mathrm{W}\right)$ near the town of Richard Toll in the Saint-Louis region in the Senegal River Basin (Fig. 1). The Richard Toll/Lac de Guiers area has undergone significant modifications such as desalination and creation of irrigation canals with permanent changes to local ecology, favoring expansion of snail intermediate host habitats, and increased sharing of water contact points by communities with their animals. In Barkedji, temporary ponds are an important source of water for human populations and their animals. These ephemeral water sources disappear completely during the dry season, interrupting transmission of schistosomiasis and necessitating seasonal migration by a large proportion of livestock-keeping communities. In both studied areas water contact points are used simultaneously by people and their livestock encouraging the transmission of schistosomiasis between and within humans and animals [21]. In the area of Lac de Guiers human schistosomiasis prevalence in humans can be as high as $88 \%$ and $47 \%$ in Barkedji [9]. In Senegal, S. bovis, S. curassoni, and hybrids of S. bovis:S. curassoni are the prevalent species causing livestock schistosomiasis [6, 12]. Recent work of Léger et al. [21] on livestock schistosomiasis in Lac de Guiers and Barkedji areas found that $S$. bovis is the primary species causing livestock schistosomiasis in Lac de Guiers area is $S$. bovis and $S$. curassoni in the Barkedji one. The prevalence estimates in slaughtered animals in the two regions were as high as $85 \%$ for Lac de Guiers and 92\% for Barkedji [21].

\section{Study overview}

First, a generic partial budget model for the estimation of disease costs was conceptualised and data needs identified based on knowledge of the effects of livestock schistosomiasis and variables commonly used in impact studies of livestock disease. Subsequently, protocols were developed for a cross-sectional interview-based survey and focus group discussions with farmers covering questions on knowledge, occurrence and manifestations of livestock schistosomiasis, herd and production data as well as management of livestock and disease.

The data collected were analysed and used to develop and parameterise specific production and partial budget models for the two sites and to define scenarios in line with local production and management practices. Secondary data and expert opinion were collated to complement the primary data where needed. Finally, livestock schistosomiasis disease costs were estimated for herds or flocks of cattle, sheep and goats using stochastic simulations in RiskAmp add-in for Excel with 10,000 iterations for a time frame of one year.

\section{Primary data collection and use Participant selection}

Target participants were subsistence and transhumance livestock farmers, i.e. the predominant ruminant production system in the two regions, rearing cattle, sheep and/or goats whose livestock produce are consumed by their households 
or they sell to neighbours/at the local market. The selling of animals or products often takes place on a needs basis to cover expenditures such as school fees; if there is no need, assets are commonly stored in the form of the herd or flock.

\section{Data collection and analysis}

From the twelve villages selected from Barkedji and the Lac de Guiers regions, eight of them had participated previously in the ZELS project and four villages (two in each region) were newly recruited. For the cross-sectional survey, questions were encoded in Open Data Kit (ODK) mobile data collection software. The questionnaire covered the following topics: demographics, production and management practices (including disease management and selling of animals and products), impact of livestock deaths on livelihood, prevention behaviour in people and animals, knowledge of disease in humans and livestock, signs of the disease in livestock and equity tool; most questions were closed and a few were open. The full survey questionnaire details are available upon request from the corresponding author(s). Each survey participant was also asked to complete a table about the number of animals owned per species, age group (young, adult), sex, and breed (local, exotic or cross-bred). The survey was translated from English to French and administered by local enumerators following a training session with the researchers leading the field work.

Farmers who participated in the survey were also invited to participate in focus group discussions (FGDs) to gather data on general signs of animal disease, signs of schistosomiasis in livestock, selling and buying of animals, milk and meat, feed and medicine including prices. All group activities were facilitated by a local enumerator with one person acting as note-taker; the language used was Wolof. The full question guide can be found in supplementary information 1 . Summary notes were generated, and the discussions were recorded in full. The recordings were transcribed and then translated by the Senegalese research collaborators into English.

Data were collected in August and September 2019. Upon completion of the survey, data were downloaded from ODK and stored as an Excel file on a safe RVC drive. The tables on livestock numbers were collected as hard copies and manually added to the Excel file using the identifier code given to each participant. The translated transcripts of the FGDs were sent to the research team based at the RVC for storage and analysis.

\section{Consent and ethical approval}

For all primary data collection activities, the researchers first explained what the study was about, how the data collection would work and the rights of the participants. Following that, each participant was asked to give their consent, which was either recorded as oral or written consent in the survey software or as written consent for the FGDs. Ethical approval was sought and granted by the i) Clinical Research and Ethical Review Board at the Royal Veterinary College; approval numbers URN 20151327 and 2019 1899-3; and (ii) the Comité National d’Ethique pour la Recherche en Santé (Dakar, Senegal) application SEN15/68.

\section{Data cleaning and analysis}

Survey data were checked for completeness and cleaned which entailed mainly harmonisation of spelling in open question fields. Answers available in French in the open comment fields were translated to English by the authors and professional translators. Data on the demographics of respondents, knowledge on schistosomiasis and economic impact of the disease were analysed. Microsoft Excel was used to calculate summary statistics and to visualize the data. For uncertain variables (e.g. those with skewed distribution, inconsistent or too few responses) probability distributions were assigned to the input variables. The open questions were read in detail in the search of information that would be relevant for the conceptualisation of the economic models including the definition of scenarios; relevant information was extracted as summary statements. For example, some respondents stated that sick animals in the herd will lose value and condition and explained a need to replace them with new ones; this informed the replacement strategy used in building the models. Data about why livestock were kept, milking animals with schistosomiasis, which animals are sold and bought were extracted from individual interviews. All FGDs were analysed for questions on daily feed quantity and type of feed

Page 5/31 
consumed by animals, cost of feed, farmers selling sick animals or not and questions on whether animals with schistosomiasis sell differently. Common topics were identified across responses for the FGDs and interviews which were used to inform the structure of the partial budget model and the input variables.

\section{Estimation of the financial impact of livestock schistosomiasis Model development and scenarios}

Stochastic models were developed in Microsoft Excel with RiskAmp add-in for simulation modelling; they are available on request from the corresponding author. Programme Evaluation and Review Technique (PERT) distribution was assigned to the identified uncertain parameters. The information gained from the analysis of the primary data collected, available literature and expert opinion was used to decide on what species to include, and to define scenarios for the financial impact analysis. The data were used to model a representative herd or flock for each species including the number of animals per age group and sex. Further, the information was used to define scenarios for the analysis.

Integrated production and partial budget analysis models were set up for one year, which is approximately the production cycle of lactating cows in the study populations. Two scenarios were considered based on the most common practices reported by respondents. Scenario 1 was a situation where farmers would test and treat their animals when seeing clinical signs consistent with livestock schistosomiasis. Scenario 2 was a situation where farmers would not test and treat their animals when seeing schistosomiasis in their herds or flocks. The detailed scenario description is given in Table 1. 
Table 1

Definition of scenarios. Sick animals are animals with clinical signs.

\begin{tabular}{|c|c|c|c|c|}
\hline & $\begin{array}{l}\text { Scenario 1: Farmers who consult } \\
\text { veterinarians and test for } \\
\text { schistosomiasis in their animals }\end{array}$ & $\begin{array}{l}\text { Scenario 2: Farmers who } \\
\text { do not consult } \\
\text { veterinarians or test or } \\
\text { treat their animals }\end{array}$ & Reasoning & $\begin{array}{l}\text { Information } \\
\text { source for } \\
\text { reasoning }\end{array}$ \\
\hline $\begin{array}{l}\text { Testing } \\
\text { strategy }\end{array}$ & $\begin{array}{l}\text { A defined proportion of sick } \\
\text { animals will get tested }\end{array}$ & No sick animal gets tested & $\begin{array}{l}\text { Not all farmers } \\
\text { test the animals. } \\
\text { Those who have } \\
\text { health-seeking } \\
\text { behaviour might } \\
\text { not be able to } \\
\text { afford the cost of } \\
\text { testing of sick } \\
\text { animals }\end{array}$ & $\begin{array}{l}\text { Primary } \\
\text { data: } \\
\text { Survey }\end{array}$ \\
\hline $\begin{array}{l}\text { Treatment } \\
\text { strategy }\end{array}$ & $\begin{array}{l}\text { A defined proportion of tested } \\
\text { animals will get treated and a } \\
\text { defined proportion of untested } \\
\text { animals will get treated. }\end{array}$ & $\begin{array}{l}\text { No sick animals get } \\
\text { treated for } \\
\text { schistosomiasis }\end{array}$ & $\begin{array}{l}\text { Not all farmers } \\
\text { who test can } \\
\text { afford the } \\
\text { treatment costs } \\
\text { for all the animals. } \\
\text { Not all farmers } \\
\text { can afford the } \\
\text { treatment costs } \\
\text { for all sick } \\
\text { animals. }\end{array}$ & $\begin{array}{l}\text { Primary } \\
\text { data: } \\
\text { Survey }\end{array}$ \\
\hline $\begin{array}{l}\text { Effectiveness } \\
\text { of treatment }\end{array}$ & $\begin{array}{l}\text { The sick animals that get treated } \\
\text { with Praziquantel recover } \\
\text { following the treatment }\end{array}$ & Not applicable & $\begin{array}{l}\text { Praziquantel is the } \\
\text { medical treatment } \\
\text { most commonly } \\
\text { used and it is } \\
\text { known to be } \\
\text { effective }\end{array}$ & $\begin{array}{l}\text { Primary } \\
\text { data: } \\
\text { Survey; } \\
\text { literature } \\
\text { [35] }\end{array}$ \\
\hline $\begin{array}{l}\text { Replacement } \\
\text { strategy }\end{array}$ & $\begin{array}{l}\text { Treated animals will recover and } \\
\text { not be replaced. The majority of } \\
\text { untreated sick animals, } \\
\text { irrespective of age, will be sold at } \\
\text { a lower market price. A proportion } \\
\text { of the animals sold will be } \\
\text { replaced with the same type of } \\
\text { animal (young for young, adult for } \\
\text { adult). }\end{array}$ & $\begin{array}{l}\text { The majority of sick } \\
\text { animals, irrespective of } \\
\text { age, will be sold at a lower } \\
\text { market price. A proportion } \\
\text { of the animals sold will be } \\
\text { replaced with the same } \\
\text { type of animal (young for } \\
\text { young, adult for adult) }\end{array}$ & $\begin{array}{l}\text { Sick animals in } \\
\text { the herd will lose } \\
\text { value and } \\
\text { condition, hence } \\
\text { the need to replace } \\
\text { them with new } \\
\text { ones }\end{array}$ & $\begin{array}{l}\text { Primary } \\
\text { data: Group } \\
\text { discussion }\end{array}$ \\
\hline $\begin{array}{l}\text { Feed and } \\
\text { supplement } \\
\text { quantity }\end{array}$ & $\begin{array}{l}\text { No change in feed and } \\
\text { supplement quantity for sick } \\
\text { animals. }\end{array}$ & $\begin{array}{l}\text { No change in feed and } \\
\text { supplement quantity for } \\
\text { sick animals. }\end{array}$ & $\begin{array}{l}\text { There will not be } \\
\text { an increase in feed } \\
\text { quantity for sick } \\
\text { animals, but they } \\
\text { will lose condition, } \\
\text { because of the } \\
\text { higher energy } \\
\text { requirement. }\end{array}$ & $\begin{array}{l}\text { Primary } \\
\text { data: Group } \\
\text { discussion } \\
\text { and survey; } \\
\text { expert } \\
\text { opinion }\end{array}$ \\
\hline $\begin{array}{l}\text { Milk yield } \\
\text { and lactation } \\
\text { duration }\end{array}$ & $\begin{array}{l}\text { Sick animals will have a reduction } \\
\text { in milk yield and a shorter } \\
\text { lactation period compared to } \\
\text { healthy females }\end{array}$ & $\begin{array}{l}\text { Sick animals will have a } \\
\text { reduction in milk yield and } \\
\text { a shorter lactation period } \\
\text { compared to healthy } \\
\text { females }\end{array}$ & $\begin{array}{l}\text { Animals that are } \\
\text { sick because of } \\
\text { schistosomiasis } \\
\text { have a lower milk } \\
\text { yield and a shorter } \\
\text { lactation period }\end{array}$ & $\begin{array}{l}\text { Literature: } \\
{[13,33]}\end{array}$ \\
\hline
\end{tabular}

\section{Partial budget analysis}

The financial impact per year was the net value calculated for each species and scenario using this basic equation:

Net value $=($ Costs saved + Added revenue $)-($ New costs + Revenue forgone $) E q .1$ 
Each of the six models (two scenarios per species, three species in total) had distinct input parameters as listed in Tables 2 and 3. 
Table 2

Input variables used to estimate disease cost - general parameters

\begin{tabular}{|c|c|c|c|c|c|c|c|}
\hline Variable & Unit & Notation & $\begin{array}{l}\text { Value } \\
\text { for } \\
\text { cattle }\end{array}$ & $\begin{array}{l}\text { Value } \\
\text { for } \\
\text { sheep }\end{array}$ & $\begin{array}{l}\text { Value } \\
\text { for } \\
\text { goats }\end{array}$ & Explanation & Reference \\
\hline $\begin{array}{l}\text { Proportion of } \\
\text { lactating } \\
\text { females } \\
\text { among adult } \\
\text { animals }\end{array}$ & $\%$ & $P_{L F}$ & 0.53 & 0.63 & 0.63 & $\begin{array}{l}\text { Estimated based on survey } \\
\text { data considering the ratio of } \\
\text { dry to lactating animals and } \\
\text { the median proportion of } \\
\text { female animals in a herd }\end{array}$ & Survey \\
\hline $\begin{array}{l}\text { Number of } \\
\text { young } \\
\text { animals }\end{array}$ & Heads & $\mathrm{N}_{Y}$ & 6 & 26 & 26 & \multirow[t]{2}{*}{$\begin{array}{l}\text { Information provided by the } \\
\text { respondents }\end{array}$} & \multirow[t]{2}{*}{ Survey } \\
\hline $\begin{array}{l}\text { Number of } \\
\text { adult } \\
\text { animals }\end{array}$ & Heads & $\mathrm{N}_{\mathrm{A}}$ & 16 & 35 & 35 & & \\
\hline $\begin{array}{l}\text { Morbidity } \\
\text { rate in young } \\
\text { animals }\end{array}$ & year $_{1}^{-}$ & $\mathrm{Mb}_{\mathrm{Y}}$ & $\begin{array}{l}\text { Pert } \\
(0.017, \\
0.021, \\
0.25)\end{array}$ & $\begin{array}{l}\text { Pert } \\
(0.1 \\
0.125, \\
0.15)\end{array}$ & $\begin{array}{l}\text { Pert } \\
(0.1 \\
0.125, \\
0.15)\end{array}$ & \multirow[t]{2}{*}{$\begin{array}{l}\text { High morbidity rate due to the } \\
\text { reported high prevalence of } \\
\text { schistosomiasis in the } \\
\text { regions. }\end{array}$} & \multirow[t]{2}{*}{$\begin{array}{l}\text { Expert } \\
\text { opinion and } \\
\text { literature }\end{array}$} \\
\hline $\begin{array}{l}\text { Morbidity } \\
\text { rate in adult } \\
\text { animals }\end{array}$ & year $_{1}^{-}$ & $\mathrm{Mb}_{\mathrm{A}}$ & $\begin{array}{l}\text { Pert } \\
(0.017 \\
0.021 \\
0.25)\end{array}$ & $\begin{array}{l}\text { Pert } \\
(0.1 \\
0.125 \\
0.15)\end{array}$ & $\begin{array}{l}\text { Pert } \\
(0.1 \\
0.125 \\
0.15)\end{array}$ & & \\
\hline $\begin{array}{l}\text { Average } \\
\text { duration of } \\
\text { clinical } \\
\text { illness if } \\
\text { animal } \\
\text { treated } \\
\text { (days) }\end{array}$ & d & $\mathrm{D}_{\mathrm{CIT}}$ & 7.00 & 7.00 & 7.00 & $\begin{array}{l}\text { When praziquantel is used, } \\
\text { then the animals will improve } \\
\text { within a few days, as } \\
\text { parasites start dying very } \\
\text { soon. }\end{array}$ & Assumption \\
\hline $\begin{array}{l}\text { Average } \\
\text { duration of } \\
\text { clinical } \\
\text { illness if } \\
\text { animal not } \\
\text { treated } \\
\text { (days) }\end{array}$ & $d$ & $\mathrm{D}_{\mathrm{Cl}}$ & 183.0 & 183.0 & 183.0 & $\begin{array}{l}\text { When animals are not treated, } \\
\text { they will not recover and be } \\
\text { continually ill. Infection and } \\
\text { clinical illness could start at } \\
\text { the beginning of the year or } \\
\text { anytime throughout. Here, a } \\
\text { mid-year infection and } \\
\text { subsequent clinical illness is } \\
\text { assumed. }\end{array}$ & Assumption \\
\hline $\begin{array}{l}\text { Average } \\
\text { duration of } \\
\text { lactation in } \\
\text { healthy } \\
\text { females }\end{array}$ & $d$ & $\mathrm{D}_{\mathrm{LF}}$ & 270.0 & 260.0 & 260.0 & $\begin{array}{l}\text { In cows, average duration for } \\
\text { lactation in Senegal is } 210- \\
270 \text { days ( } 7 \text { to } 9 \text { months); the } \\
\text { majority of respondents } \\
\text { reported a lactation duration } \\
\text { of } 6 \text { to } 12 \text { months. Therefore, } \\
\text { the } 9 \text { months value was } \\
\text { chosen.In dams, average } \\
\text { duration for lactation is } 260 \\
\text { days according to existing } \\
\text { literature. }\end{array}$ & $\begin{array}{l}\text { Literature } \\
{[32,36,37]} \\
\text { and survey }\end{array}$ \\
\hline
\end{tabular}




\begin{tabular}{|c|c|c|c|c|c|c|c|}
\hline Variable & Unit & Notation & $\begin{array}{l}\text { Value } \\
\text { for } \\
\text { cattle }\end{array}$ & $\begin{array}{l}\text { Value } \\
\text { for } \\
\text { sheep }\end{array}$ & $\begin{array}{l}\text { Value } \\
\text { for } \\
\text { goats }\end{array}$ & Explanation & Reference \\
\hline $\begin{array}{l}\text { Average } \\
\text { duration } \\
\text { without the } \\
\text { animals sold } \\
\text { and not } \\
\text { replaced in } \\
\text { the } \\
\text { herd/flock }\end{array}$ & d & $D_{S}$ & 183.0 & 183.0 & 183.0 & $\begin{array}{l}\text { It is assumed that animals } \\
\text { are sold mid-year and will } \\
\text { therefore not be present in the } \\
\text { herd or flock for half of the } \\
\text { year }\end{array}$ & Assumption \\
\hline $\begin{array}{l}\text { Daily milk } \\
\text { quantity in } \\
\text { healthy } \\
\text { female }\end{array}$ & I & $\mathrm{M}_{\mathrm{HA}}$ & $\begin{array}{l}\text { Pert } \\
(1.0,2, \\
3.5)\end{array}$ & $\begin{array}{l}\text { Pert } \\
(0.5, \\
1.0,1.2)\end{array}$ & $\begin{array}{l}\text { Pert } \\
(0.5, \\
1.0,1.2)\end{array}$ & $\begin{array}{l}\text { Median values from survey } \\
\text { used as a basis }\end{array}$ & Survey \\
\hline $\begin{array}{l}\text { Daily } \\
\text { concentrate } \\
\text { feed quantity } \\
\text { in healthy } \\
\text { animals }\end{array}$ & $\mathrm{kg}$ & $\mathrm{F}_{\mathrm{HA}}$ & $\begin{array}{l}\text { Pert } \\
(3.0, \\
4.0,5.0)\end{array}$ & $\begin{array}{l}\text { Pert } \\
(0.8,1, \\
1.2)\end{array}$ & $\begin{array}{l}\text { Pert } \\
(0.8,1, \\
1.2)\end{array}$ & $\begin{array}{l}\text { Value mentioned most often } \\
\text { in group discussion }\end{array}$ & FGD \\
\hline $\begin{array}{l}\text { Daily } \\
\text { supplement } \\
\text { quantity in } \\
\text { healthy } \\
\text { animals }\end{array}$ & $\mathrm{kg}$ & $\mathrm{S}_{\mathrm{HA}}$ & $\begin{array}{l}\text { Pert } \\
(0.8 \\
0.1 \\
0.12)\end{array}$ & $\begin{array}{l}\text { Pert } \\
(0, \\
0.025, \\
0.04)\end{array}$ & $\begin{array}{l}\text { Pert } \\
(0, \\
0.025, \\
0.04)\end{array}$ & Value from literature & Literature \\
\hline $\begin{array}{l}\text { Market price } \\
\text { for young } \\
\text { healthy } \\
\text { animal }\end{array}$ & XOF & $\mathrm{Pr}_{\mathrm{YHA}}$ & 270,000 & 36,250 & 26,250 & $\begin{array}{l}\text { Information provided by the } \\
\text { respondents }\end{array}$ & FGD \\
\hline $\begin{array}{l}\text { Market price } \\
\text { for young } \\
\text { sick animal }\end{array}$ & XOF & $\mathrm{Pr}_{\mathrm{YSA}}$ & 200,000 & 30,000 & 20,000 & & \\
\hline $\begin{array}{l}\text { Market price } \\
\text { for adult } \\
\text { healthy } \\
\text { animal }\end{array}$ & XOF & $\mathrm{Pr}_{\mathrm{AHA}}$ & 380,000 & 40,000 & 38,000 & & \\
\hline $\begin{array}{l}\text { Market price } \\
\text { for adult sick } \\
\text { animal }\end{array}$ & XOF & $\mathrm{Pr}_{\mathrm{ASA}}$ & 330,000 & 38,000 & 30,000 & & \\
\hline $\begin{array}{l}\text { Price of milk } \\
\text { per litre for } \\
\text { healthy } \\
\text { animals }\end{array}$ & XOF & $\operatorname{Pr}_{\mathrm{MHA}}$ & 557.92 & 601.70 & 530.88 & & \\
\hline $\begin{array}{l}\text { Price of milk } \\
\text { per litre for } \\
\text { sick animals }\end{array}$ & XOF & $\mathrm{Pr}_{\mathrm{MSA}}$ & 525.00 & 500.00 & 500.00 & $\begin{array}{l}\text { Information provided by the } \\
\text { respondents and triangulated } \\
\text { with survey data from [38] }\end{array}$ & $\begin{array}{l}\text { FGD and } \\
{[38]}\end{array}$ \\
\hline $\begin{array}{l}\text { Price of } \\
\text { concentrate } \\
\text { feed per kg }\end{array}$ & XOF & $\operatorname{Pr}_{F}$ & $\begin{array}{l}\text { Pert } \\
(34.6 \\
43 \\
51.9)\end{array}$ & $\begin{array}{l}\text { Pert } \\
(34.6 \\
43 \\
51.9)\end{array}$ & $\begin{array}{l}\text { Pert } \\
(34.6 \\
43 \\
51.9)\end{array}$ & $\begin{array}{l}\text { Price of feed as reported by } \\
\text { respondents }\end{array}$ & Survey \\
\hline $\begin{array}{l}\text { Price of } \\
\text { supplement } \\
\text { per kg }\end{array}$ & XOF & $\mathrm{Pr}_{\mathrm{S}}$ & 50.00 & 452.00 & 452.00 & $\begin{array}{l}\text { Calculated based on data } \\
\text { from [38] }\end{array}$ & $\begin{array}{l}\text { Literature } \\
{[38]}\end{array}$ \\
\hline
\end{tabular}




\begin{tabular}{|c|c|c|c|c|c|c|c|}
\hline Variable & Unit & Notation & $\begin{array}{l}\text { Value } \\
\text { for } \\
\text { cattle }\end{array}$ & $\begin{array}{l}\text { Value } \\
\text { for } \\
\text { sheep }\end{array}$ & $\begin{array}{l}\text { Value } \\
\text { for } \\
\text { goats }\end{array}$ & Explanation & Reference \\
\hline $\begin{array}{l}\text { Price of } \\
\text { testing per } \\
\text { animal }\end{array}$ & XOF & $\mathrm{Pr}_{\mathrm{Te}}$ & 1525 & $\begin{array}{l}\text { Pert } \\
(75.24 \\
83.6 \\
91.96)\end{array}$ & $\begin{array}{l}\text { Pert } \\
(75.24 \\
83.6 \\
91.96)\end{array}$ & $\begin{array}{l}\text { Information provided by the } \\
\text { respondents }\end{array}$ & Survey, FGD \\
\hline $\begin{array}{l}\text { Price of } \\
\text { routine } \\
\text { treatment per } \\
\text { animal }\end{array}$ & XOF & $\mathrm{Pr}_{\mathrm{T}}$ & $\begin{array}{l}\text { Pert }( \\
18,23 \\
28)\end{array}$ & $\begin{array}{l}\text { Pert ( } \\
18,23 \\
28)\end{array}$ & $\begin{array}{l}\text { Pert ( } \\
18,23 \\
28)\end{array}$ & $\begin{array}{l}\text { Medical expenditure for } \\
\text { animals in a herd that } \\
\text { include, e.g. vaccination, } \\
\text { deworming, tick treatment }\end{array}$ & $\begin{array}{l}\text { Survey and } \\
\text { [38] }\end{array}$ \\
\hline $\begin{array}{l}\text { Price of } \\
\text { clinical } \\
\text { treatment per } \\
\text { animal (for } \\
\text { veterinary } \\
\text { use } \\
\text { praziquantel) }\end{array}$ & XOF & $\mathrm{P}_{\mathrm{Tr}}$ & $\begin{array}{l}\text { Pert } \\
\text { (510.35, } \\
567.05 \\
623.76)\end{array}$ & $\begin{array}{l}\text { Pert } \\
\text { (510.35, } \\
567.05 \\
623.76)\end{array}$ & $\begin{array}{l}\text { Pert } \\
(510.35 \\
567.05 \\
623.76)\end{array}$ & $\begin{array}{l}\text { Price of a praziquantel tablet } \\
\text { for animals that have clinical } \\
\text { disease caused by } \\
\text { schistosomiasis }\end{array}$ & $\begin{array}{l}\text { Literature } \\
\text { [39] }\end{array}$ \\
\hline
\end{tabular}


Table 3

Input variables used to estimate disease cost - scenario specific parameters

\begin{tabular}{|c|c|c|c|c|c|c|c|c|}
\hline \multirow[b]{2}{*}{ Variable } & \multirow[b]{2}{*}{ Unit } & \multirow[b]{2}{*}{ Notation } & \multicolumn{2}{|l|}{ Cattle } & \multicolumn{4}{|c|}{ Sheep and goats } \\
\hline & & & $\begin{array}{l}\text { Scenario } \\
1\end{array}$ & $\begin{array}{l}\text { Scenario } \\
2\end{array}$ & $\begin{array}{l}\text { Scenario } \\
1\end{array}$ & $\begin{array}{l}\text { Scenario } \\
2\end{array}$ & Explanation & Reference \\
\hline $\begin{array}{l}\text { Average } \\
\text { duration } \\
\text { of clinical } \\
\text { illness } \\
\text { before } \\
\text { animal is } \\
\text { sold }\end{array}$ & $\mathrm{d}$ & $\mathrm{D}_{\mathrm{CIS}}$ & 14.0 & 7.0 & 14.0 & 7.0 & $\begin{array}{l}\text { Number of days } \\
\text { animals stay in the } \\
\text { herd/flock before } \\
\text { being sold; this } \\
\text { reflects the } \\
\text { observation and } \\
\text { decision time of } \\
\text { the farmer. It is } \\
\text { assumed that they } \\
\text { will observe the } \\
\text { animal to see if it } \\
\text { gets better and } \\
\text { then sell it. It is } \\
\text { also assumed that } \\
\text { those sold are sold } \\
\text { early to get a better } \\
\text { market price, when } \\
\text { they still have } \\
\text { some condition. }\end{array}$ & Assumption \\
\hline $\begin{array}{l}\text { Proportion } \\
\text { of sick } \\
\text { animals } \\
\text { tested }\end{array}$ & $\%$ & $\mathrm{P}_{\mathrm{TS}}$ & $\begin{array}{l}\text { Pert } \\
(0.24 \\
0.31 \\
0.36)\end{array}$ & 0.00 & $\begin{array}{l}\text { Pert } \\
(0.24, \\
0.3, \\
0.36)\end{array}$ & 0.00 & $\begin{array}{l}\text { Only a handful of } \\
\text { the animals } \\
\text { showing clinical } \\
\text { signs will be } \\
\text { tested. Those who } \\
\text { have health- } \\
\text { seeking behaviour } \\
\text { might not be able } \\
\text { to afford the cost } \\
\text { of testing for all } \\
\text { sick animals }\end{array}$ & $\begin{array}{l}\text { Assumption } \\
\text { based on } \\
\text { [13] }\end{array}$ \\
\hline $\begin{array}{l}\text { Proportion } \\
\text { of tested } \\
\text { animals } \\
\text { that get } \\
\text { treated }\end{array}$ & $\%$ & $\mathrm{P}_{\mathrm{TT}}$ & $\begin{array}{l}\text { Pert } \\
\\
(0.40 \\
0.50 \\
0.60)\end{array}$ & 0.00 & $\begin{array}{l}\text { Pert } \\
\\
(0.40 \\
0.50 \\
0.60)\end{array}$ & 0.00 & $\begin{array}{l}\text { Not all farmers } \\
\text { who test will be } \\
\text { able to afford the } \\
\text { treatment costs for } \\
\text { all the animals, } \\
\text { hence only some } \\
\text { will treat }\end{array}$ & Assumption \\
\hline $\begin{array}{l}\text { Proportion } \\
\text { of } \\
\text { untested } \\
\text { animals } \\
\text { that get } \\
\text { treated }\end{array}$ & $\%$ & $P_{\text {UTT }}$ & $\begin{array}{l}\text { Pert } \\
(0.64, \\
0.80 \\
0.96)\end{array}$ & 0.00 & $\begin{array}{l}\text { Pert } \\
(0.64, \\
0.80 \\
0.96)\end{array}$ & 0.00 & $\begin{array}{l}\text { It is assumed that } \\
\text { some farmers with } \\
\text { health-seeking } \\
\text { behaviour will treat } \\
\text { some of the sick } \\
\text { animals, either } \\
\text { they were tested or } \\
\text { not }\end{array}$ & Assumption \\
\hline
\end{tabular}




\begin{tabular}{|c|c|c|c|c|c|c|c|c|}
\hline \multirow[b]{2}{*}{$\begin{array}{l}\text { Proportion } \\
\text { of sick } \\
\text { animals } \\
\text { sold } \\
\text { among } \\
\text { those not } \\
\text { treated }\end{array}$} & \multirow[b]{2}{*}{$\%$} & \multirow[b]{2}{*}{$P_{S}$} & \multicolumn{2}{|l|}{ Cattle } & \multicolumn{2}{|c|}{ Sheep and goats } & \multirow[b]{2}{*}{$\begin{array}{l}\text { Farmers reported } \\
\text { in the survey that } \\
\text { they sell all types } \\
\text { of animals (young, } \\
\text { adult, old, } \\
\text { production, and } \\
\text { breeding animals). } \\
\text { Many also } \\
\text { indicated to sell } \\
\text { animals when they } \\
\text { are sick. It is } \\
\text { assumed that } \\
\text { farmers sell most } \\
\text { of the sick animals } \\
\text { that they }\end{array}$} & \multirow[b]{2}{*}{$\begin{array}{l}\text { Survey and } \\
\text { assumption }\end{array}$} \\
\hline & & & $\begin{array}{l}\text { Pert } \\
(0.90 \\
0.95 \\
1.00)\end{array}$ & $\begin{array}{l}\text { Pert } \\
(0.90 \\
0.95 \\
1.00)\end{array}$ & $\begin{array}{l}\text { Pert } \\
(0.90 \\
0.95 \\
1.00)\end{array}$ & $\begin{array}{l}\text { Pert } \\
(0.90 \\
0.95 \\
1.00)\end{array}$ & & \\
\hline $\begin{array}{l}\text { Proportion } \\
\text { of sick } \\
\text { animals } \\
\text { sold that } \\
\text { are } \\
\text { replaced }\end{array}$ & $\%$ & $P_{\text {SAR }}$ & $\begin{array}{l}\text { Pert } \\
(0.56 \\
0.70 \\
0.84)\end{array}$ & $\begin{array}{l}\text { Pert } \\
(0.40 \\
0.50 \\
0.60)\end{array}$ & $\begin{array}{l}\text { Pert } \\
(0.56, \\
0.70 \\
0.84)\end{array}$ & $\begin{array}{l}\text { Pert } \\
(0.40 \\
0.50 \\
0.60)\end{array}$ & $\begin{array}{l}\text { Because farmers } \\
\text { like to maintain } \\
\text { their herds (their } \\
\text { asset), it is } \\
\text { assumed that a } \\
\text { proportion of the } \\
\text { animals sold will } \\
\text { be replaced. } \\
\text { Because farmers } \\
\text { in scenario } 2 \text { have } \\
\text { more animals to } \\
\text { sell, their } \\
\text { replacement rate is } \\
\text { lower, as they will } \\
\text { not have the } \\
\text { means to replace } \\
\text { so many animals. }\end{array}$ & Assumption \\
\hline $\begin{array}{l}\text { Rate of } \\
\text { reduced } \\
\text { lactation } \\
\text { duration } \\
\text { in sick } \\
\text { females } \\
\text { (due to } \\
\text { disease) }\end{array}$ & year $_{1}^{-}$ & $\mathrm{R}_{\mathrm{LF}}$ & $\begin{array}{l}\text { Pert } \\
(0.032 \\
0.04 \\
0.048)\end{array}$ & $\begin{array}{l}\text { Pert } \\
(0.032 \\
0.04 \\
0.048)\end{array}$ & $\begin{array}{l}\text { Pert } \\
(0.10, \\
0.12, \\
0.15)\end{array}$ & $\begin{array}{l}\text { Pert } \\
(0.10 \\
0.12 \\
0.15)\end{array}$ & $\begin{array}{l}\text { The lactation } \\
\text { duration of sick } \\
\text { females will be } \\
\text { shortened. }\end{array}$ & $\begin{array}{l}\text { Assumption } \\
\text { based on } \\
\text { literature } \\
{[13,40]}\end{array}$ \\
\hline $\begin{array}{l}\text { Rate of } \\
\text { reduced } \\
\text { milk yield } \\
\text { in sick } \\
\text { cows (due } \\
\text { to } \\
\text { disease) }\end{array}$ & ${ }_{1}^{\text {year }^{-}}$ & $\mathrm{R}_{\mathrm{MY}}$ & $\begin{array}{l}\text { Pert } \\
(0.08 \\
0.1 \\
0.12)\end{array}$ & $\begin{array}{l}\text { Pert } \\
(0.08 \\
0.1 \\
0.12)\end{array}$ & $\begin{array}{l}\text { Pert } \\
(0.08, \\
0.1 \\
0.12)\end{array}$ & $\begin{array}{l}\text { Pert } \\
(0.08 \\
0.1 \\
0.12)\end{array}$ & $\begin{array}{l}\text { The milk yield of } \\
\text { sick females will } \\
\text { be reduced. }\end{array}$ & Survey, FGD \\
\hline $\begin{array}{l}\text { Mortality } \\
\text { rate } \\
\text { young } \\
\text { animal } \\
\text { among } \\
\text { those sick } \\
\text { and not } \\
\text { treated }\end{array}$ & year $_{1}^{-}$ & $\mathrm{Mt}_{\mathrm{Y}}$ & $\begin{array}{l}\text { Pert } \\
(0.032 \\
0.04 \\
0.048)\end{array}$ & $\begin{array}{l}\text { Pert } \\
(0.032 \\
0.04 \\
0.048)\end{array}$ & $\begin{array}{l}\text { Pert } \\
(0.07 \\
0.10 \\
0.13)\end{array}$ & $\begin{array}{l}\text { Pert } \\
(0.07 \\
0.10 \\
0.13)\end{array}$ & $\begin{array}{l}\text { Information by } \\
\text { respondents and } \\
\text { expert opinion. } \\
\text { There is very little } \\
\text { mortality caused } \\
\text { by } \\
\text { schistosomiasis. } \\
\text { Additional } \\
\text { mortality due to } \\
\text { schistosomiasis in } \\
\text { cattle/sheep/goats } \\
\text { is not perceptible } \\
\text { in regular } \\
\text { production years }\end{array}$ & $\begin{array}{l}\text { Survey, } \\
\text { expert } \\
\text { opinion }\end{array}$ \\
\hline
\end{tabular}




\begin{tabular}{|c|c|c|c|c|c|c|}
\hline \multirow[b]{2}{*}{$\begin{array}{l}\text { Mortality } \\
\text { rate adult } \\
\text { animal } \\
\text { among } \\
\text { those sick } \\
\text { and not } \\
\text { treated }\end{array}$} & \multirow[b]{2}{*}{${ }_{1}^{\text {year }}$} & \multirow[b]{2}{*}{$\mathrm{Mt}_{\mathrm{A}}$} & \multicolumn{2}{|l|}{ Cattle } & \multicolumn{2}{|c|}{ Sheep and goats } \\
\hline & & & $\begin{array}{l}\text { Pert } \\
(0.032, \\
0.04 \\
0.048)\end{array}$ & $\begin{array}{l}\text { Pert } \\
(0.032, \\
0.04 \\
0.048)\end{array}$ & $\begin{array}{l}\text { Pert } \\
(0.07, \\
0.10, \\
0.13)\end{array}$ & $\begin{array}{l}\text { Pert } \\
(0.07, \\
0.10, \\
0.13)\end{array}$ \\
\hline
\end{tabular}

New costs were additional costs for testing and treatment and replacement of sick animals.

For scenario 1, this included costs of

Testing young sick animals $=\mathrm{N}_{\mathrm{Y}} * \mathrm{Mb}_{\mathrm{Y}} * \mathrm{P}_{\mathrm{TS}} * \mathrm{Pr}_{\mathrm{Te}}$ Eq. 2

Where $N_{Y}$ stands for the number of young animals, $M b_{Y}$ for the morbidity rate of young animals, $P_{T S}$ for the proportion of sick animals tested, and $\operatorname{Pr}_{T e}$ for the price of testing per animal.

Testing adult sick animals $=\mathrm{N}_{\mathrm{A}} * \mathrm{Mb}_{\mathrm{A}} * \mathrm{P}_{\mathrm{TS}} * \mathrm{Pr}_{\mathrm{Te}}$ Eq. 3

Where $N_{A}$ stands for the number of adult animals and $M b_{A}$ the morbidity rate of adult animals.

Treating sick animals tested $=\left(N_{A} * M b_{A}+N_{Y} * M b_{Y}\right) * P_{T S} * P_{T T} * P r_{T r}$ Eq. 4

Where $P_{T T}$ stands for the proportion of tested animals that get treated, and $\operatorname{Pr}_{T r}$ the price of treatment per animal.

Treating sick animals not tested $=\left(N_{A} * M b_{A}+N_{Y} * M b_{Y}\right) *\left(1-P_{T S}\right) * P_{U T T} * \operatorname{Pr}_{T r} E q .5$

Where $P_{U T T}$ stands for the proportion of untested animals treated.

For scenarios 1 and 2, this included costs of

Replacing sick animals $=\left(N_{A} * M b_{A} * P r_{A H A}+N_{Y} * M b_{Y} * P r_{Y H A}\right) *\left[P_{T S} *\left(1-P_{T T}\right)+\left(1-P_{T S}\right) *\left(1-P_{U T T}\right)\right] * P_{S U T} * P_{S A R} E q .6$

Where $\operatorname{Pr}_{A H A}$ stands for the market price of adult healthy animal, $\operatorname{Pr}_{Y H A}$ the market price of young healthy animal, $P_{S U T}$ for the proportion of sick animals sold among those not treated, and $P_{S A R}$ for the proportion of young sick animals sold that are replaced.

Revenue forgone stemmed from milk not sold and selling animals at a lower market value. For scenarios 1 and 2 , this included revenue forgone from

Milk not sold from sick cows (not sold) due to shortened lactation $=N_{A} * M b_{A} * P_{L F} *\left[P_{T S} *\left(1-P_{T T}\right)+\left(1-P_{T S}\right) *\left(1-P_{U T T}\right)\right] *$ $\left(1-P_{S}\right) * R_{L F} * D_{C l} * M_{H A} * \operatorname{Pr}_{M H A} E q .7$

Where $P_{L F}$ stands for the proportion of lactating females among the adult animals, $P_{S}$ for proportion of sick animals sold, $R_{L F}$ for the rate of reduced lactation days, $D_{C l}$ the duration of clinical illness if an animal is not treated, $M_{H A}$ the daily milk quantity in healthy animals, and $\operatorname{Pr}_{M H A}$ the price of milk per litre for a healthy animal.

Milk not sold from sick cows (not sold) due to reduced milk production per day $=N_{A} * M b_{A} * P_{L F} *\left[P_{T S} *\left(1-P_{T T}\right)+\left(1-P_{T S}\right)\right.$ *(1- $\left.\left.P_{U T T}\right)\right] *\left(1-P_{S}\right) * D_{C l} * R_{M Y} * M_{H A} * \operatorname{Pr}_{M H A} E q .8$

Where $R_{M Y}$ is the rate of reduced milk yield in sick cows. 
Milk not sold from sick cows (not sold) due to reduced milk price $=N_{A} * M b_{A} * P_{L F} *\left[P_{T S} *\left(1-P_{T T}\right)+\left(1-P_{T S}\right) *\left(1-P_{U T T}\right)\right]$ * $\left(1-P_{S}\right) * D_{C l} * R_{M Y} * M_{H A} *\left(\operatorname{Pr}_{M H A}-\operatorname{Pr}_{M S A}\right)$ Eq. 9

Where $\operatorname{Pr}_{M S A}$ is the price of milk per litre for a sick animal.

Milk not sold from sick cows before they are being sold $=N_{A} * M b_{A} * P_{L F} *\left[P_{T S} *\left(1-P_{T T}\right)+\left(1-P_{T S}\right) *\left(1-P_{U T T}\right)\right] * P_{S} * D_{C I S}$ * $R_{M Y} * M_{H A} *\left(\operatorname{Pr}_{M H A}-\operatorname{Pr}_{M S A}\right)$ Eq. 10

Where $D_{C I S}$ is the average duration of clinical illness before animal is sold.

Sick animals sold at lower market price $=\left[N_{A} * M b_{A} *\left(\operatorname{Pr}_{A H A}-\operatorname{Pr}_{A S A}\right)+N_{Y} * M b_{Y} *\left(\operatorname{Pr}_{Y H A}-P r_{Y S A}\right)\right] *\left[P_{T S} *\left(1-P_{T T}\right)+\left(1-P_{T S}\right.\right.$ )$\left.*\left(1-P_{U T T}\right)\right] * P_{S} E q .11$

Where $\operatorname{Pr}_{A S A}$ stands for the market price of an adult sick animal and $\operatorname{Pr}_{A S A}$ for the market price of a young sick animal.

Value reduction of animals not sold (but alive) $=\left[N_{A} * M b_{A} *\left(\operatorname{Pr}_{A H A}-\operatorname{Pr}_{A S A}\right) *\left(1-M t_{A}\right)+N_{Y} * M b_{Y} *\left(\operatorname{Pr}_{Y H A}-\operatorname{Pr}_{Y S A}\right)\right] *(1-$ $\left.M t_{Y}\right) *\left[P_{T S} *\left(1-P_{T T}\right)+\left(1-P_{T S}\right) *\left(1-P_{U T T}\right)\right] *\left(1-P_{S}\right) E q .12$

Where $M t_{A}$ and $M t_{Y}$ are the mortality rate for adult and young animals, respectively, among those sick and not sold.

Herd value reduction due to sick animals sold and not replaced $=\left(N_{A} * M b_{A} * \operatorname{Pr}_{A H A}+N_{Y} * M b_{Y} * \operatorname{Pr}_{Y H A}\right) *\left[P_{T S} *\left(1-P_{T T}\right)+\right.$ $\left.\left(1-P_{T S}\right) *\left(1-P_{U T T}\right)\right] * P_{S} *\left(1-P_{S A R}\right) E q .13$

Value reduction of sick, untreated animals not sold and dead $=\left(N_{A} * M b_{A} * \operatorname{Pr}_{A H A} * M t_{A}+N_{Y} * M b_{Y} * \operatorname{Pr}_{Y H A} * M t_{Y}\right) *\left[P_{T S}\right.$ * $\left.\left(1-P_{T T}\right)+\left(1-P_{T S}\right) *\left(1-P_{U T T}\right)\right] *\left(1-P_{S}\right) E q .14$

Expenditures saved stemmed from saving concentrate feed, supplements and routine treatment. For scenarios 1 and 2 , this included expenditures saved from

Concentrate feed saved on sick animals sold and not replaced $=\left(N_{A} * M b_{A} * \operatorname{Pr}_{A H A}+N_{Y} * M b_{Y} * \operatorname{Pr}_{Y H A}\right) *\left[P_{T S} *\left(1-P_{T T}\right)+\right.$ $\left.\left(1-P_{T S}\right) *\left(1-P_{U T T}\right)\right] * P_{S U T} *\left(1-P_{S A R}\right) * D_{S} * F_{H A} * \operatorname{Pr}_{F} E q .15$

Where $D_{S}$ stands for the number of days without the animals in the herd/flock, $F_{H A}$ the daily concentrate feed quantity in $\mathrm{kg}$ in healthy animals, and $\operatorname{Pr}_{F}$ the concentrate feed per $\mathrm{kg}$.

Concentrate feed saved on untreated animals not sold and dead $=\left(N_{A} * M b_{A} * M t_{A}+N_{Y} * M b_{Y} * M t_{Y}\right) *\left[P_{T S} *\left(1-P_{T T}\right)+(1-\right.$ $\left.\left.P_{T S}\right) *\left(1-P_{U T T}\right)\right] *\left(1-P_{S}\right) * D_{S} * F_{H A} * P_{F} E q .16$

Supplement saved on sick animals sold and not replaced $=\left(N_{A} * M b_{A} * \operatorname{Pr}_{A H A}+N_{Y} * M b_{Y} * \operatorname{Pr}_{Y H A}\right) *\left[P_{T S} *\left(1-P_{T T}\right)+(1-\right.$ $\left.\left.P_{T S}\right) *\left(1-P_{U T T}\right)\right] * P_{S U T} *\left(1-P_{S A R}\right) * D_{S} * S_{H A} * P_{S U} E q .17$

Where $S_{H A}$ stands for daily supplement quantity in $\mathrm{kg}$ in healthy animals and $P_{S u}$ the supplement price per $\mathrm{kg}$.

Supplement saved on untreated animals not sold and dead $=\left(N_{A} * M b_{A} * M t_{A}+N_{Y} * M b_{Y} * M t_{Y}\right) *\left[P_{T S} *\left(1-P_{T T}\right)+\left(1-P_{T S}\right)\right.$ *(1- $\left.\left.P_{U T T}\right)\right] *\left(1-P_{S}\right) * D_{S} * S_{H A} * P_{S U} E q .18$

Routine treatment saved on sick animals sold and not replaced $=\left(N_{A} * M b_{A} * \operatorname{Pr}_{A H A}+N_{Y} * M b_{Y} * \operatorname{Pr}_{Y H A}\right) *\left[P_{T S} *\left(1-P_{T T}\right)+\right.$ $\left.\left(1-P_{T S}\right) *\left(1-P_{U T T}\right)\right] * P_{S U T} *\left(1-P_{S A R}\right) * D_{S} * P_{R T} E q .19$

Where $\operatorname{Pr}_{R T}$ stands for the price of routine treatment per animal per day. 
Routine treatment saved on untreated animals not sold and dead $=\left(N_{A} * M b_{A} * M t_{A}+N_{Y} * M b_{Y} * M t_{Y}\right) *\left[P_{T S} *\left(1-P_{T T}\right)+\right.$ $\left.\left(1-P_{T S}\right) *\left(1-P_{U T T}\right)\right] *\left(1-P_{S}\right) * D_{S} * \operatorname{Pr}_{R T} E q .20$

Extra revenue consisted in the revenue from selling young sick animals:

Revenue from young sick animals sold due to disease $=\left(N_{A} * M b_{A} * \operatorname{Pr}_{A S A}+N_{Y} * M b_{Y} * \operatorname{Pr}_{Y S A}\right) *\left[P_{T S} *\left(1-P_{T T}\right)+\left(1-P_{T S}\right) *\right.$ $\left.\left(1-P_{U T T}\right)\right] * P_{S} E q .21$

The partial budget models did not consider the effect on labour, as these production systems rely predominantly on unpaid family labour. All prices used for the models were in Senegalese currency - West African CFA franc; 1 XOF $=0$.

0014 GBP as at the time of analysis (2020). Each PBA model was run with 10,000 iterations, the net values were assigned as outputs. Finally, the impact of uncertain variables on the output of models (net value) was conducted using the in-built function performing univariate regression analysis.

\section{Results}

\section{Respondent demographics}

A total of 92 respondents representing different households participated in the survey; demographic characteristics are shown in Table 4. 
Demographic characteristics of respondents, $n=92$

\begin{tabular}{|c|c|}
\hline Characteristic & Number (percentage) \\
\hline Gender & $71(77)$ \\
\hline Male & $21(23)$ \\
\hline Female & $7(8)$ \\
\hline Age & $20(22)$ \\
\hline Below 20 years & $22(24)$ \\
\hline $21-30$ years & $21(23)$ \\
\hline $31-40$ years & $16(17)$ \\
\hline $41-50$ years & $6(7)$ \\
\hline $51-60$ years & $11(12)$ \\
\hline Above 60 years & $9(10)$ \\
\hline Location & $9(10)$ \\
\hline Mayel (Barkedji) & $8(9)$ \\
\hline Didjiery (Richard Toll) & $8(9)$ \\
\hline Loumbel Mbada (Linguere) & $8(9)$ \\
\hline Medina Cheikhou (Lac de Guiers) & $8(9)$ \\
\hline Ndombo (Lac de Guiers) & $8(9)$ \\
\hline Pathe Badio (Lac de Guiers) & $7(8)$ \\
\hline Barkedji (Linguere) & $6(7)$ \\
\hline Mbane (Lac de Guiers) & $6(7)$ \\
\hline Loumbel Lana (Linguere) & $4(4)$ \\
\hline Ngao (Linguere) & $66(71)$ \\
\hline Ngassama (Linguere) & $34(37)$ \\
\hline Mourseyni (Lac de Guiers) & $25(27)$ \\
\hline Occupation & $6(7)$ \\
\hline Livestock merchant & $3(3)$ \\
\hline Farmer & $1(1)$ \\
\hline Merchant & $1(1)$ \\
\hline Housewife & $58(63)$ \\
\hline Student & $13(14)$ \\
\hline Teacher & $11(12)$ \\
\hline Health worker & $2(2)$ \\
\hline Source of income & $1(1)$ \\
\hline Breeding & $1(1)$ \\
\hline
\end{tabular}




\begin{tabular}{|ll|}
\hline CCharăacteristic & Number (percentage) \\
\hline Agriculture & $1(1)$ \\
Livestock sales & $1(1)$ \\
Agriculture and breeding & $1(1)$ \\
Breeding and fishing & $2(2)$ \\
Dependent on parents & \\
Fishing & \\
Student & \\
Teaching & \\
Not mentioned & \\
\hline
\end{tabular}

\section{Production and disease management}

In both study areas, the most predominant breed of animals in all species were local breeds. Cattle was regarded by survey respondents as the most important livestock (49\% respondents), followed by sheep ( $27 \%$ of respondents) and then goats ( $5 \%$ of respondents). Local, cross and exotic breeds of all three species were kept in the two study areas (supplementary information 2). The animals were mostly kept for dual production type such as meat and breeding, dairy and breeding or meat and dairy; and the triple combination of meat, dairy and breeding. In the predominant breed, i.e., local breed, cattle, sheep and goats were kept mostly for triple-purpose of meat, dairy and breeding $(41 \%, 34 \%$, and $35 \%$ respectively) and dual-purpose of dairy and breeding (30\%,22\%, $15 \%$ respectively). With regards to the treatment of animals, $57 / 92$ respondents (62\%) stated that they routinely treated their animals per year. A total of $84 / 92$ respondents (91\%) stated that they routinely gave their animals supplements.

\section{Signs of schistosomiasis in animals and schistosomiasis related management practices}

A total of $81 / 92$ respondents (88\%) reported they knew that animals can be infected with schistosomiasis, while 11 of them (12\%) reported not knowing. The most common signs of schistosomiasis reported by respondents for cattle, sheep, and goats are displayed in Table 5 . A total of 48/92 respondents (52\%) reported that they would seek advice from local veterinary workers if they thought their livestock had schistosomiasis; 33/92 respondents (36\%) had never tested their livestock in the past for schistosomiasis and 28/92 (85\%) used a veterinary clinic. With regards to treatment, 35/92 respondents (38\%) of respondents stated that they have treated their livestock in the last four years for schistosomiasis with 33/92 respondents (36\%) using "Tenicure" (PZQ-Levamisole combination) to treat. 
Table 5

Signs of schistosomiasis as reported by respondents in the survey

\begin{tabular}{|c|c|}
\hline Signs in cattle & $\begin{array}{l}\text { Number (percentage) } \\
\mathrm{n}=\mathbf{8 1}\end{array}$ \\
\hline Weight loss & $52(64)$ \\
\hline Hollowing around eye & $52(64)$ \\
\hline Diarrhoea & $28(35)$ \\
\hline Weakness & $20(25)$ \\
\hline Blood in urine & $12(15)$ \\
\hline Blood in stool & $10(12)$ \\
\hline Abortion & $3(4)$ \\
\hline Dehydration & $2(2)$ \\
\hline Don't know & $9(11)$ \\
\hline Signs in sheep & $\begin{array}{l}\text { Number (percentage) } \\
\mathrm{n}=20\end{array}$ \\
\hline Hollowing around eye & $13(65)$ \\
\hline Weight loss & $11(55)$ \\
\hline Diarrhoea & $11(55)$ \\
\hline Blood in urine & $7(35)$ \\
\hline Blood in stool & $4(20)$ \\
\hline Weakness & $4(20)$ \\
\hline Abortion & $2(10)$ \\
\hline Dehydration & $1(5)$ \\
\hline Don't know & $2(10)$ \\
\hline Signs in goats & $\begin{array}{l}\text { Number (percentage) } \\
\mathrm{n}=71\end{array}$ \\
\hline Weight loss & $48(68)$ \\
\hline Hollowing around eye & $45(63)$ \\
\hline Diarrhoea & $21(30)$ \\
\hline Weakness & $13(18)$ \\
\hline Blood in stool & $9(13)$ \\
\hline Blood in urine & $8(11)$ \\
\hline Abortion & $2(3)$ \\
\hline Dehydration & $1(1)$ \\
\hline Don't know & $9(13)$ \\
\hline
\end{tabular}




\section{Net disease costs estimated using partial budget analysis}

Results for livestock schistosomiasis costs per animal and year in the species studied are shown in Tables 6, 7 and 8. For cattle, the median net disease value for a standard cattle herd with 22 animals were XOF - 13,408 (min - 45,508; max + 10,808) for Scenario 1 and XOF - 49,296 ( $\min$ - 141,972; $\max +32,246$ ) for Scenario 2. For sheep, the median net costs of disease for a standard sheep flock with 61 animals were XOF - 27,227 ( $\min -82,423$; max + 16,483) for Scenario 1 and XOF - 70,072 ( $\min -219,980 ; \max +80,956$ ) for Scenario 2. For goats, the median net costs of disease for a standard goat herd with 61 animals were XOF - 27,694 ( $\min -76,654$; $\max +7,048)$ for Scenario 1 and XOF - 70,281 (min - 196,835; max $+60,321)$ for Scenario 2. In all models, the largest contribution to the total impact was caused by replacing of animals, herd value reduction, and revenue from young sick animals sold due to disease. 
Table 6

Livestock schistosomiasis disease costs in XOF for a common cattle herd in Senegal considering two scenarios.

\begin{tabular}{|c|c|c|c|}
\hline Costs & Scenario 1 & & $\begin{array}{l}\text { Scenario } \\
2\end{array}$ \\
\hline \multirow{5}{*}{$\begin{array}{l}\text { Additional } \\
\text { expenditures }\end{array}$} & Testing of young sick animals & 44 & - \\
\hline & Testing of adult sick animals & 109 & - \\
\hline & Treatment for sick animals tested & 45 & - \\
\hline & Treatment for sick animals not tested & 161 & - \\
\hline & Replacing animals sold & 23903 & 83740 \\
\hline \multirow[t]{8}{*}{$\begin{array}{l}\text { Revenue } \\
\text { foregone }\end{array}$} & $\begin{array}{l}\text { Milk not sold from sick cows because lactation duration is shortened by a } \\
\text { certain number of days }\end{array}$ & 66 & 108 \\
\hline & $\begin{array}{l}\text { Milk not sold from sick cows kept in herd because of reduced milk } \\
\text { production per day }\end{array}$ & 168 & 297 \\
\hline & Milk not sold from sick cows kept in herd because of reduced milk price & 10 & 18 \\
\hline & Milk not sold from sick cows before they are being sold & 12 & 28 \\
\hline & $\begin{array}{l}\text { Revenue foregone on sick animals sold by selling at lower market value } \\
\text { (price reduction) }\end{array}$ & 6560 & 29116 \\
\hline & Value reduction of animals not sold (but alive) & 388 & 3763 \\
\hline & Herd value reduction because of the sick animals sold and NOT replaced & 13460 & 82096 \\
\hline & Value reduction of sick, untreated animals not sold and dead & 84 & 161 \\
\hline Total of costs & & 45010 & 199327 \\
\hline \multicolumn{4}{|l|}{ Benefits } \\
\hline & Concentrate feed saved on sick animals sold and not replaced & 1190 & 7255 \\
\hline & Concentrate feed saved on sick, untreated animals not sold and dead & 8 & 14 \\
\hline & Supplement saved on sick animals sold and not replaced & - & - \\
\hline & Supplement saved on sick, untreated animals not sold and dead & - & - \\
\hline & Routine treatment saved on sick animals sold and not replaced & 164 & 1000 \\
\hline & Routine treatment saved on sick, untreated animals not sold and dead & 1 & 2 \\
\hline Extra revenue & Revenue from young sick animals sold due to disease & 30803 & 136720 \\
\hline Total of benefits & & 32166 & 144992 \\
\hline \multirow{4}{*}{$\begin{array}{l}\text { Net disease } \\
\text { costs }\end{array}$} & Mean & -13729 & -49476 \\
\hline & Median & -13408 & -49296 \\
\hline & Min & -45508 & -141972 \\
\hline & Max & +10808 & +32246 \\
\hline
\end{tabular}


Table 7

Livestock schistosomiasis disease costs in XOF for a common sheep flock in Senegal considering two scenarios.

\begin{tabular}{|c|c|c|c|}
\hline Costs & Item & $\begin{array}{l}\text { Scenario } \\
1\end{array}$ & $\begin{array}{l}\text { Scenario } \\
2\end{array}$ \\
\hline \multirow{5}{*}{$\begin{array}{l}\text { Additional } \\
\text { expenditures }\end{array}$} & Testing of young sick animals & 100 & - \\
\hline & Testing of adult sick animals & 132 & - \\
\hline & Treatment for sick animals tested & 845 & - \\
\hline & Treatment for sick animals not tested & 2411 & - \\
\hline & Replacing animals sold & 54527 & 148649 \\
\hline \multirow[t]{8}{*}{$\begin{array}{l}\text { Revenue } \\
\text { foregone }\end{array}$} & $\begin{array}{l}\text { Milk not sold from sick sheep because lactation duration is shortened by } \\
\text { a certain number of days }\end{array}$ & 1734 & 4968 \\
\hline & $\begin{array}{l}\text { Milk not sold from sick sheep kept in herd because of reduced milk } \\
\text { production per day }\end{array}$ & 1247 & 4443 \\
\hline & Milk not sold from sick sheep kept in herd because of reduced milk price & 211 & 751 \\
\hline & Milk not sold from sick sheep before they are being sold & 164 & 333 \\
\hline & $\begin{array}{l}\text { Revenue foregone on sick animals sold by selling at lower market value } \\
\text { (price reduction) }\end{array}$ & 8744 & 29587 \\
\hline & Value reduction of animals not sold (but alive) & 442 & 12046 \\
\hline & Herd value reduction because of the sick animals sold and NOT replaced & 27357 & 128426 \\
\hline & Value reduction of sick, untreated animals not sold and dead & 3928 & 11827 \\
\hline Total of costs & & 101841 & 341031 \\
\hline \multicolumn{4}{|l|}{ Benefits } \\
\hline & Concentrate feed saved on sick animals sold and not replaced & 7021 & 32962 \\
\hline & Concentrate feed saved on sick, untreated animals not sold and dead & 1007 & 3044 \\
\hline & Supplement saved on sick animals sold and not replaced & 1379 & 6474 \\
\hline & Supplement saved on sick, untreated animals not sold and dead & 198 & 598 \\
\hline & Routine treatment saved on sick animals sold and not replaced & 3369 & 15816 \\
\hline & Routine treatment saved on sick, untreated animals not sold and dead & 483 & 1461 \\
\hline Extra revenue & Revenue from young sick animals sold due to disease & 73140 & 247488 \\
\hline Total of benefits & & 86598 & 307843 \\
\hline \multirow{4}{*}{$\begin{array}{l}\text { Net disease } \\
\text { costs }\end{array}$} & Mean & -28042 & -69894 \\
\hline & Median & -27227 & -70072 \\
\hline & Min & -82423 & $\overline{2} 19980$ \\
\hline & Max & +16483 & +80956 \\
\hline
\end{tabular}


Table 8

Livestock schistosomiasis disease costs in XOF for a common goat herd in Senegal considering two scenarios.

\begin{tabular}{|c|c|c|c|}
\hline Costs & Item & $\begin{array}{l}\text { Scenario } \\
1\end{array}$ & $\begin{array}{l}\text { Scenario } \\
2\end{array}$ \\
\hline \multirow{5}{*}{$\begin{array}{l}\text { Additional } \\
\text { expenditures }\end{array}$} & Testing of young sick animals & 72 & - \\
\hline & Testing of adult sick animals & 108 & - \\
\hline & Treatment for sick animals tested & 601 & - \\
\hline & Treatment for sick animals not tested & 2275 & - \\
\hline & Replacing animals sold & 47246 & 109949 \\
\hline \multirow[t]{8}{*}{$\begin{array}{l}\text { Revenue } \\
\text { foregone }\end{array}$} & $\begin{array}{l}\text { Milk not sold from sick goats because lactation duration is shortened by } \\
\text { a certain number of days }\end{array}$ & 1650 & 3640 \\
\hline & $\begin{array}{l}\text { Milk not sold from sick goats kept in herd because of reduced milk } \\
\text { production per day }\end{array}$ & 1541 & 2941 \\
\hline & Milk not sold from sick goats kept in herd because of reduced milk price & 90 & 171 \\
\hline & Milk not sold from sick goats before they are being sold & 83 & 125 \\
\hline & $\begin{array}{l}\text { Revenue foregone on sick animals sold by selling at lower market value } \\
\text { (price reduction) }\end{array}$ & 10010 & 34742 \\
\hline & Value reduction of animals not sold (but alive) & 408 & 5252 \\
\hline & Herd value reduction because of the sick animals sold and NOT replaced & 16907 & 112715 \\
\hline & Value reduction of sick, untreated animals not sold and dead & 2690 & 6397 \\
\hline Total of costs & & 83682 & 275931 \\
\hline \multicolumn{4}{|l|}{ Benefits } \\
\hline & Concentrate feed saved on sick animals sold and not replaced & 3961 & 26409 \\
\hline & Concentrate feed saved on sick, untreated animals not sold and dead & 629 & 1495 \\
\hline & Supplement saved on sick animals sold and not replaced & 1283 & 8551 \\
\hline & Supplement saved on sick, untreated animals not sold and dead & 204 & 484 \\
\hline & Routine treatment saved on sick animals sold and not replaced & 2083 & 13888 \\
\hline & Routine treatment saved on sick, untreated animals not sold and dead & 331 & 786 \\
\hline Extra revenue & Revenue from young sick animals sold due to disease & 54144 & 187922 \\
\hline Total of benefits & & 62634 & 239535 \\
\hline \multirow{4}{*}{$\begin{array}{l}\text { Net disease } \\
\text { costs }\end{array}$} & Mean & -28282 & -70144 \\
\hline & Median & -27694 & -70281 \\
\hline & Min & -76654 & $-\overline{196835}$ \\
\hline & Max & +7048 & +60321 \\
\hline
\end{tabular}

Sensitivity analyses showed that the market prices for young and adult, healthy and sick animals had the biggest impact on the net value for all species with the highest regression coefficients for the market price for adult healthy animals 
( 0.355 to 0.542$)$ followed by the market price for adult sick animals ( 0.253 to 0.381$)$, the market price for young healthy animals (0.039 to 0.180$)$, and the market price for young sick animals ( 0.016 to 0.099$)$, daily feed quantity, rate of reduced feed intake and the rate of reduced lactation (regression coefficients between 0.01 and 0.03 ). The proportion of untested animals that get treated also had a big influence on the net value in scenario 1 with regression coefficients of 0.092 for goats, 0.069 for sheep, and 0.067 for cattle. The morbidity rate in adult animals had regression coefficients of 0.019 (scenario 1, goats), 0.013 (scenario 2, goats), and 0.011 (scenario 1, sheep); the morbidity rate in young animals in goats had a regression coefficient of 0.012 . The variable sick animals sold that are replaced had regression coefficients of 0.021 (scenario 1, goats) and 0.013 (scenario 1, sheep). The other uncertain variables all had regression coefficients $<0.01$.

\section{Discussion}

In this study the financial impact of livestock schistosomiasis on livestock keepers in two regions of Senegal was shown to be substantial; particularly in scenario 2 . The median disease costs in a representative herd for the areas studied amounted to 0.23 to 1.22 of an average monthly income for people living in rural Senegal is XOF 57,461 [41] with the disease costs highest in small ruminants. Thus, having schistosomiasis in a herd will reduce the farmers' livelihoods and, in some instances, potentially cause a situation where basic needs cannot be covered anymore.

The costs were highest in scenario 2, i.e., a situation where farmers do not test and treat animals. The survey data showed that farmers test for schistosomiasis, but no information was available on the specific diagnostic test(s) used by the veterinary technicians in the study areas. Because of the existing practice of selling sick animals, the financial impact estimated was caused mainly by the selling and buying of animals and changes in herd value. With weight loss being a prominent sign of schistosomiasis infection reported by respondents, sick animals fetch a lower market price and cause replacement costs for the farmer. Consequently, farmers have an interest in selling sick, untreated animals soon to avoid a further reduction in market price. With the clinical signs reported including weight loss, hollowing around the eye, and diarrhoea, sick animals are likely recognised as such by potential buyers and they will only pay the price for a sick animal. Because the subsistence and transhumance farmers studied sell animals only based on needs and usually maintain their herd of flock size as a capital asset, the reduction in herd value was modelled explicitly.

In partial budget models for farming units operating on a commercial basis, i.e., where products are sold to make profits, the change in herd value is not commonly incorporated in a partial budget [42, 43]. However, in a setting where the herd or flock is not used as a means to make profit, but has the function of a social and capital asset, the estimation of its change in value appears justified. Using the models described, the loss in herd value was a major cost to the farmers; caused mainly by a reduction in animals, as it was assumed that not all animals could be replaced. This was also reflected in the sensitivity analysis where the market prices of animals were shown to have the biggest influence on the financial impact. Because farmers not testing and treating will have a larger number of sick animals (than those that test and treat), but most likely will not have the means to replace all the animals they are selling, the financial impact for them was highest. This indicates that testing and treatment of animals has the potential to reduce the financial impact of livestock schistosomiasis in these populations.

A previously published study on rural development and poverty reduction reported that most people in Senegal contribute $50 \%$ of their family labour to subsistence livestock farming, which accounts for a $23.8 \%$ share of their average income [44]. Many of the respondents from the two study areas associated disease in their livestock as a big economic loss. As these farmers place great importance on their livestock, it is not surprising that some of the farmers would test as well as treat, although the cost of the diagnostic test (XOF 1,050) is higher than the medication for the disease. The costs of schistosomiasis treatment (XOF 567) seem to be affordable, yet many farmers were not testing or treating their animals. Farmers who do not test and treat, could experience a range of constraints and have other economic priorities. Kauppinen and colleagues [45], for example, reported in a study on the attitudes of farmers to animal welfare that most farmers 
considered their welfare and that of their animals as being dependent on each other. Though the farmers are aware that their animals can be infected with schistosomiasis, they may not understand that treating the animals also confers protection on them by also potentially interrupting the zoonotic transmission of the disease from animals to humans and preventing hybridization of species. Thus, further studies may need to look in more depth at the health-seeking behaviour and farmers motivation for disease control.

The accessibility to the drug and the lack of alternative drugs for livestock means that farmers may use donated PZQ intended only for human use to treat their livestock. As a consequence, a systematic mis-, and particular under-dosing of the drug in the animals can be identified as one of the factors which has led to the reported high prevalence of livestock schistosomiasis in the regions examined [9]. This is a One Health concern as the use and cross- or mis-use of PZQ in animals have been reported to potentiate resistance and reduced efficacy of the drug $[10,14,46,47,48]$. The People's Republic of China has already employed vaccine development for zoonotic $S$. japonicum in some regions, in addition controlled PZQ treatment of bovines, setting the pace for an integrated approach to schistosomiasis, simultaneously combining mitigation measures in animals with control measures in humans as part of its national control programme [49]. China's prevalence of schistosomiasis in humans and bovines is now less than 1\% [50] and if countries in Africa were to follow the Chinese example, the estimated high prevalence in humans and animals is envisaged to decline.

Importantly, the current study models the financial impact of livestock schistosomiasis on a representative herd or flock in the study areas. It is based on common practices as reported by farmers and can be seen as a common situation in a regular production year, where there are no major droughts, epidemic outbreaks or similar. Consequently, the models only capture a narrow set of infinite possibilities of impact defined by a diverse set of farmers, practices, circumstances, seasonal and annual fluctuations (caused by weather, celebrations, festive periods, etc). Further, the input values are based on a wide range of sources and assumptions, as the primary data collected did not cover all aspects sufficiently. For example, limitations were encountered when asking questions about herd size, during which several farmers seemed to give inconsistent answers. This was likely because talking about herd size is taboo based on the belief that talking about it may attract bad luck. This was also found in other studies; for example, Parisse encountered a similar problem of receiving inconsistent or approximate numbers with regards to herd sizes [ $\left.{ }^{5} 1\right]$.

The respondents in this current study were transhumant subsistence farmers who hardly ever kept records. For instance, the mortality rate could not be determined as the farmers gave no answer to this question or they were inconsistent. Similarly, the effect on feed use remained inconclusive. The milk yield produced with and without schistosomiasis could not be determined accurately, as respondents did not usually measure the quantity of milk their animals produced or that the household consumed. We also recognized that Fasciola could be a confounding factor in the diagnosis of the disease as many of the farmers reported signs that are attributable to liver fluke and other diseases. To address these limitations in input parameters, other sources were consulted including related studies, scientific literature and expert opinion. Moreover, sensitivity analyses were conducted to assess the influence on uncertain parameters on the financial impact. It is recommended for future research efforts to make an investment in the generation of baseline data for livestock populations in Senegalese transhumant and subsistence populations; these could be based on longitudinal studies looking at production, economic, social and management parameters. There seems to be a general lack of studies of production and economic studies in these settings; a problem most likely exacerbated by a shortage of animal health and One Health economists in the region that could generate knowledge on herd and production data, effects of schistosomiasis in livestock, and health-seeking behaviour. This shortage of capability and capacity will need longer-term investment in education, research and development.

Schistosomiasis is a disease that has a dual burden on human and animal health, and several studies have suggested the role the environment plays in the transmission and hybridization of the species $[16,52,53]$. A more holistic analysis of the impacts of the disease using One Health economics is recommended in the future to assess the monetary and nonmonetary impacts on animals' health. Being able to evaluate the net cost of the disease to all sectors calculating the 
separable costs for the human health and veterinary sectors, estimating the cost-benefit analysis (CBA) of an integrated intervention such as treating livestock schistosomiasis and analysing the zoonotic disability-adjusted life year (zDALY) are practical methods to evaluate the disease costs for zoonotic diseases [54].

The current study highlighted the financial impact livestock schistosomiasis has on traditional subsistence and transhumance farmers keeping cattle, sheep or goats in Northern Senegal. The presence of disease and its effects underscore the need to consider livestock schistosomiasis in control programmes. Since the benefits reaped from the treatment of livestock zoonotic infections also spill over into public health and medical sectors, though at a cost to the agricultural sector, multisectoral collaboration will be needed.

\section{Declarations}

\section{Ethics approval and consent to participate}

For all primary data collection activities, the researchers first explained what the study was about, how the data collection would work and the rights of the participants. Following that, each participant was asked to give their consent, which was either recorded as oral consent in the survey software or as written consent for the FGDs.

Ethical approval was sought and granted by the Clinical Research and Ethical Review Board at the Royal Veterinary College; approval number URN 2019 1899-3.

\section{Consent for publication}

Not applicable

\section{Availability of data and materials}

All data generated or analysed during this study are included in this published article [and its supplementary information files]. Other datasets used and/or analysed can be made available by the corresponding author on reasonable request.

\section{Competing interests}

We declare no competing interests.

\section{Funding}

This work was part of the project "Control And Targeted Treatment for Livestock Emerging Schistosomiasis (CATTLES) " funded by the UK Biotechnology and Biological Sciences Research Council, the UK Department for International Development, the UK Economic and Social Research Council, the UK Medical Research Council, the UK Natural Environment Research Council, and the UK Defence Science and Technology Laboratory, under the Zoonoses and Emerging Livestock Systems (ZELS) programme (Reference BB/S013822/1; JPW [principal investigator (P) ] with BH, LW, MS and ND [co-investigators (Co-ls)]); and the project 'A multi-disciplinary approach to optimize, evaluate social uptake, and mathematically predict the impact of a novel point-of-contact diagnostic test for targeted treatment of zoonotic hybrid and livestock schistosomiasis in sub-Saharan Africa' funded by Research England: The Bloomsbury SET - Connecting Capability to Combat Infectious Disease and Antimicrobial Resistanceproject grant (Reference SET-POC; Ref CCF-17-7779; JPW (PI) with EL (co-I).

\section{Authors' contributions}

JPW, BH, EL conceptualized the study; EL, EH, LW, JPW and BH designed data collection tools; EH, SD, ND, MS performed field-work and/or facilitated access to farmers; $\mathrm{BH}$ and PA designed and performed economic data analyses. Original draft 
preparation was performed by PA, while $\mathrm{BH}$ and JPW were major contributors in writing the manuscript. All authors read and approved the final manuscript.

\section{ACKNOWLEDGEMENTS}

We thank the Senegalese communities involved in the project, the local facilitators, especially that of Mapate Gaye [resident of Richard Toll]. We are also particularly grateful to Dr Samba Diop of the Institut Supérieur de Formation Agricole et Rurale, Université de Thiès, Bambey, Senegal for his full support and assistance regarding the economic evaluations in the field, the interview team who helped with data collection and translation, comprising of Mr. Cheikh Tidiane Thiam, Mr. Farota Souleymane, Mr. Ben Abass Faye, Mr. Alassane Ndiaye, Mr. Sam Moustapha, Mr. Alioune Sy and Mr. Simon Senghor. We are also extremely grateful to Dr Chrissy Roberts from the London School of Tropical Medicine and Hygiene for full use and access throughout to their OpenDataKit (ODK) hardware and software.

We thank Drs Linda Waldman and Tabitha Hrynick from the Institute of Development Studies in the United Kingdom and Louise Vince from the Royal Veterinary College for their input into the design of the data collection protocols. We are indebted to Prof Javier Guitian, Dr Imadidden Musallam and Dr Laura Craighead from the Royal Veterinary College, and their collaborators, for giving us access to their data on Senegalese cattle production and health management systems for triangulation purposes and fill data gaps. Their data were generated by the project 'Establishment of a multi-sectoral strategy for the control of brucellosis in the main peri-urban dairy production zones of West and Central Africa', funded by the Zoonoses and Emerging Livestock Systems (ZELS), a joint research initiative between; Biotechnology and Biological Sciences Research Council (BBSRC), Defence Science and Technology Laboratory (DSTL), Department for International Development (DFID), Economic and Social Research Council (ESRC), Medical Research Council (MRC) and Natural Environment Research Council (NERC).

This study is, in part, a product of PA's master's dissertation in One Health under the supervision of BH and JPW.

\section{References}

1. Parasites - Schistosomiasis [Internet]. [Atlanta]: Centres for Disease Control; 2018 [cited 21 May 2021] Available from: https://www.cdc.gov/parasites/schistosomiasis/gen_info/faqs.html.

2. Webster BL, Diaw OT, Seye MM, Webster JP, Rollinson D. Introgressive hybridization of Schistosoma haematobium group species in Senegal: species barrier break down between ruminant and human schistosomes. PLoS Negl Trop Dis. 2013 Apr 4;7(4):e2110.

3. Colley DG, Bustinduy AL, Secor WE, King CH. Human schistosomiasis. Lancet. 2014;383:2253-64.

4. De Bont J, Vercruysse J. The epidemiology and control of cattle schistosomiasis. Parasitol Today. 1997;13(7):25562.

5. Schistosomiasis [Internet]. [Geneva]: World Health Organization; 2019a [cited 21 May 2021]. Available from: https://www.who.int/en/news-room/fact-sheets/detail/schistosomiasis [Accessed 19 October 2019].

6. Rudge JW, Webster JP, Lu DB, Wang TP, Fang GR, Basáñez MG. Identifying host species driving transmission of schistosomiasis japonica, a multihost parasite system, in China. Proc Natl Acad Sci. 2013 Jul;9(28):11457-62. 110(.

7. Gordon CA, Kurscheid J, Williams GM, Clements AC, Li Y, Zhou XN, Utzinger J, McManus DP, Gray DJ. Asian schistosomiasis: current status and prospects for control leading to elimination. Trop Med Infect Dis. 2019 Mar;4(1):40.

8. Van Dorssen CF, Gordon CA, Li Y, Williams GM, Wang Y, Luo Z, et al. Rodents, goats and dogs-their potential roles in the transmission of schistosomiasis in China. Parasitol. 2017 Oct;144(12):1633-42.

9. Léger E, Borlase A, Fall CB, Diouf ND, Diop SD, Yasenev L, Catalano S, Thiam CT, Ndiaye A, Emery A, Morrell A. Prevalence and distribution of schistosomiasis in human, livestock, and snail populations in northern Senegal: a One 
Health epidemiological study of a multi-host system. Lancet Planet Health. 2020 Aug 1;4(8):e330-42.

10. Catalano S, Léger E, Fall CB, Borlase A, Diop SD, Berger D, Webster BL, Faye B, Diouf ND, Rollinson D, Sène M. Multihost transmission of Schistosoma mansoni in Senegal, 2015-2018. Emerg Infect Dis. 2020 Jun;26(6):1234.

11. Gower CM, Vince L, Webster JP. Should we be treating animal schistosomiasis in Africa? The need for a One Health economic evaluation of schistosomiasis control in people and their livestock. Trans R Soc Trop Med Hyg. 2017 Jun 1;111(6):244-7.

12. Liang S, Ponpetch K, Zhou Y, Guo J, Erko B, Stothard JR, et al. A. Diagnosis of Schistosoma Infection in Non-Human Animal Hosts: A Systematic Review and Meta-Analysis. Preprints 2021, 2021050075 (doi: 10.20944/preprints202105.0075.v1).

13. Léger E, Garba A, Hamidou AA, Webster BL, Pennance T, Rollinson D, Webster JP. Introgressed animal schistosomes Schistosoma curassoni and S. bovis naturally infecting humans. Emerg Infect Dis. 2016 Dec;22(12):2212.

14. Huyse T, Webster BL, Geldof S, Stothard JR, Diaw OT, Polman K, Rollinson D. Bidirectional introgressive hybridization between a cattle and human schistosome species. PLoS Pathog. 2009 Sep;4(9):e1000571. 5(.

15. Boon NA, Mbow M, Paredis L, Moris P, Sy I, Maes T, Webster BL, Sacko M, Volckaert FA, Polman K, Huyse T. No barrier breakdown between human and cattle schistosome species in the Senegal River Basin in the face of hybridisation. Int J Parasitol. 2019 Dec;49(13-14)(1):1039-48.

16. Léger E, Webster JP. Hybridizations within the genus Schistosoma: implications for evolution, epidemiology and control. Parasitol. 2017 Jan;144(1):65-80.

17. Catalano S, Sène M, Diouf ND, Fall CB, Borlase A, Léger E, Bâ K, Webster JP. Rodents as natural hosts of zoonotic Schistosoma species and hybrids: an epidemiological and evolutionary perspective from West Africa. J Infect Dis. 2018 Jul 2;218(3):429 - 33.

18. Webster JP, Molyneux DH, Hotez PJ, Fenwick A. The contribution of mass drug administration to global health: past, present and future. Philos Trans R Soc Lond B Biol Sci. 2014 Jun 19;369(1645):20130434.

19. Deol AK, Fleming FM, Calvo-Urbano B, Walker M, Bucumi V, Gnandou I, Tukahebwa EM, Jemu S, Mwingira UJ, Alkohlani A, Traoré M. Schistosomiasis-assessing progress toward the 2020 and 2025 global goals. N Engl J Med. 2019 Dec;26(26):2519-28. 381(.

20. Accelerating work to overcome the global impact of neglected tropical diseases: A roadmap for implementation [Internet]. Geneva: World Health Organization; 2012 [cited 21 May 2021]. Available from: https://www.who.int/neglected_diseases/NTD_RoadMap_2012_Fullversion.pdf.

21. Schistosomiasis: progress report 2001-2011, strategic plan 2012-2020 [Internet]. Geneva: WHO Reports; 2013 [cited 21 May 2021]. Available from: https://apps.who.int/iris/bitstream/handle/10665/78074/9789241503174_eng.pdf? sequence=1\&isAllowed=y.

22. Ending the neglect to attain the Sustainable Development Goals. A road map for neglected tropical diseases 20212030 [Internet]. Geneva:World Health Organization; 2021 [cited 13 Jul 2021]. Available from: https://www.who.int/neglected_diseases/Ending-the-neglect-to-attain-the-SDGs-NTD-Roadmap.pdf.

23. Ryan W. Global Health and Societal Impacts of Schistosomiasis. Idun; 2018 [cited 25 May 2021]. Available from: https://idun.augsburg.edu/etd/381.

24. Zhou XN, Wang LY, Chen MG, Wang TP, Guo JG, Wu XH, et al. An economic evaluation of the national schistosomiasis control programme in China from 1992 to 2000. Acta Trop. 2005 Nov;96(2-3)(1):255-65.

25. Jia TW, Utzinger J, Deng Y, Yang K, Li YY, Zhu JH, et al. Quantifying quality of life and disability of patients with advanced schistosomiasis japonica. PLoS Negl Trop Dis. 2011 Feb;15(2):e966. 5(.

26. Lenk EJ, Redekop WK, Luyendijk M, Rijnsburger AJ, Severens JL. Productivity loss related to neglected tropical diseases eligible for preventive chemotherapy: a systematic literature review. PLoS Negl Trop Dis. 2016 Feb 18;10(2):e0004397.

Page 28/31 
27. Secor WE. Early lessons from schistosomiasis mass drug administration programs. F1000Research. 2015;4.

28. Bachmann G. Merck Donates one Billionth Praziquantel Tablet [Internet]. Press Release of Merck KgaA; 2020 [cited 25 May 2021]. Available from: https://www.merckgroup.com/en/news/2020-01-30-one-billionth-praziquantel-tablet.html.

29. Redekop WK, Lenk EJ, Luyendijk M, Fitzpatrick C, Niessen L, Stolk WA, et al. The socioeconomic benefit to individuals of achieving the 2020 targets for five preventive chemotherapy neglected tropical diseases. PLoS Negl Trop Dis. 2017 Jan;19(1):e0005289. 11(.

30. De Bont J, Shaw DJ, Vercruysse J. The relationship between faecal egg counts, worm burden and tissue egg counts in early Schistosoma mattheei infections in cattle. Acta Trop. 2002 Jan 1;81(1):63-76.

31. Vercruysse J, Southgate VR, Rollinson D. The epidemiology of human and animal schistosomiasis in the Senegal River Basin. Acta Trop. 1985 Sep 1;42(3):249 - 59.

32. Kassuku A, Christensen NO, Monrad J, Nansen P, Knudsen J. Epidemiological studies on Schistosoma bovis in Iringa Region, Tanzania. Acta Trop. 1986 Jun 1;43(2):153 - 63.

33. McCauley EH, Majid AA, Tayeb A. Economic evaluation of the production impact of bovine schistosomiasis and vaccination in the Sudan. Prev Vet Med. 1984 Sep 1;2(6):735 - 54.

34. Alarcon P, Häsler B, Raboisson D, Waret-Szkuta A, Corbière F, Rushton J. Application of integrated production and economic models to estimate the impact of Schmallenberg virus for various sheep production types in the UK and France. Vet Rec Open. 2013 Jul;1(1):e000036.

35. Vercruysse J, Claerebout E. Praziquantel and Epsirantel [Internet]. MSD Manual: Veterinary Manual; 2014 [cited 24 July 2021] Available from: https://www.msdvetmanual.com/pharmacology/anthelmintics/praziquantel-andepsiprantel.

36. Gueye A. Review of the livestock sector/meat and milk policies that influence them in Senegal. Food and Agriculture Organization of the United Nations (FAO) and the Economic Community of West African States (ECOWAS); 2017. Available from: http://www.fao.org/3/i5273e/i5273e.pdf.

37. Fall A, Diop M, Sandford J, Wissocq YJ, Durkin JW, Trail JC. Evaluation of the productivities of Djallonke sheep and N'Dama cattle at the Centre de Recherches Zootechniques, Kolda, Senegal. International Livestock Centre for Africa; 1982.

38. Craighead L, Chengat PB, Musallam I, Ndour AP, Ayih-Akakpo AA, Fotsac DM, et al. Brucellosis in dairy herds: Farm characteristics and practices in relation to likely adoption of three potential private-public partnership (PPP) vaccination control strategies in West and Central Africa. Transbound Emerg Dis. 2021 Apr 20.

39. Praziquantel for veterinary. use in dogs, cats, horses and livestock as anthelmintic against tapeworms [Internet]. [Place unknown]: Parasitipedia; 2021 [cited 24 July 2021]. Available from: https://parasitipedia.net/index.php? option=com_content\&view=article\&id=2500\&ltemid=2772.

40. Saad AM, Hussein MF, Dargie JD, Taylor MG. The pathogenesis of experimental Schistosoma bovis infections in Sudanese sheep and goats. J Comp Pathol. 1984 Jul 1;94(3):371 - 85.

41. Kazybayeva S, Otte J, Roland-Holst D. Livestock Production and Household Income Patterns in Rural Senegal. Rome: Food and Agricultural Organization of the United Nations; 2006.

42. Swinkels JM, Hogeveen $\mathrm{H}$, Zadoks RN. A partial budget model to estimate economic benefits of lactational treatment of subclinical Staphylococcus aureus mastitis. J Dairy Sci. 2005 Dec 1;88(12):4273-87.

43. Häsler B, Alarcon P, Raboisson D, Waret-Szkuta A, Rushton J. Integration of production and financial models to analyse the financial impact of livestock diseases: a case study of Schmallenberg virus disease on British and French dairy farms. Vet Rec Open. 2015 Jan;2(1):e000035.

44. Van den Broeck G, Maertens M. Moving up or moving out? Insights into rural development and poverty reduction in Senegal. World Dev. 2017 Nov 1;99:95-109. 
45. Kauppinen T, Vainio A, Valros A, Rita H, Vesala KM. Improving animal welfare: qualitative and quantitative methodology in the study of farmers' attitudes. Anim Welf. 2010 Nov 1;19(4):523.

46. Fenwick A, Webster JP. Schistosomiasis: challenges for control, treatment and drug resistance. Curr Opin Infect Dis. 2006 Dec 1;19(6):577-82.

47. Cioli D, Pica-Mattoccia L, Basso A, Guidi A. Schistosomiasis control: praziquantel forever?. Mol Biochem Parasitol. 2014 Jun 1;195(1):23-9.

48. Adenowo AF, Oyinloye BE, Ogunyinka BI, Kappo AP. Impact of human schistosomiasis in sub-Saharan Africa. Braz J Infect Dis. 2015 Mar;19:196-205.

49. Wang L, Utzinger J, Zhou XN. Schistosomiasis control: experiences and lessons from China. Lancet. 2008 Nov 22;372(9652):1793-5.

50. Cao ZG, Zhao YE, Willingham AL, Wang TP. Towards the elimination of schistosomiasis japonica through control of the disease in domestic animals in the People's Republic of China: a tale of over 60 years. Adv Parasitol. 2016 Jan 1;92:269-306.

51. Parisse M. Developing local dairy production: the Laiterie du Berger, Senegal. Field Actions Science Reports. Field Actions Sci Rep. 2012 Feb 27;6.

52. Ndione RA, Diop D, Riveau G, Ba CT, Jouanard N. Role of environmental parameters on the density of intermediate host snails of human schistosoma during the year in the commune of Richard-Toll, Senegal. Med Sante Trop. 2018 May 1;28(2):158 - 64 .

53. Wood CL, Sokolow SH, Jones IJ, Chamberlin AJ, Lafferty KD, Kuris AM, Jocque M, Hopkins S, Adams G, Buck JC, Lund AJ. Precision mapping of snail habitat provides a powerful indicator of human schistosomiasis transmission. Proc Natl Acad Sci. 2019 Nov 12;116(46):23182-91.

54. Shaw AP, Rushton J, Roth F, Torgerson PR. DALYs, dollars and dogs: how best to analyse the economics of controlling zoonoses. Rev Sci Tech. 2017;36(1):147-61.

\section{Figures}




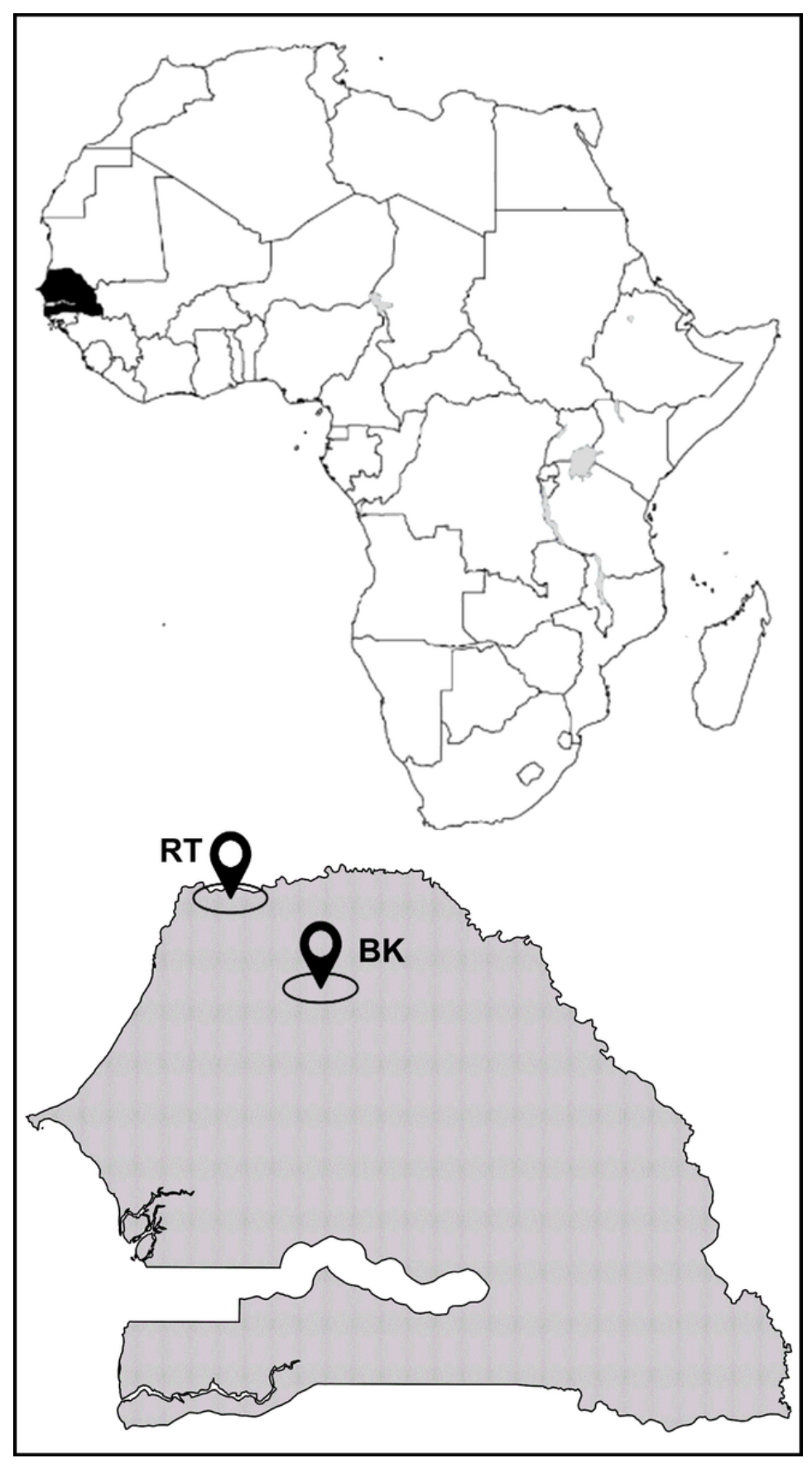

Figure 1

Map of the two study sites; Source: Modified based on [8]

\section{Supplementary Files}

This is a list of supplementary files associated with this preprint. Click to download.

- Graphicalabstract.png

- SupplementaryInformation.docx 